\title{
Control of Dynamic Hopf Bifurcations
}

\author{
N. Berglund \\ Weierstraß-Institut für Angewandte Analysis und Stochastik \\ Mohrenstraße 39, D-10117 Berlin, Germany
}

March 30, 1999

\begin{abstract}
The slow passage through a Hopf bifurcation leads to the delayed appearance of large amplitude oscillations. We construct a smooth scalar feedback control which suppresses the delay and causes the system to follow a stable equilibrium branch. This feature can be used to detect in time the loss of stability of an ageing device. As a by-product, we obtain results on the slow passage through a bifurcation with double zero eigenvalue, described by a singularly perturbed cubic Liénard equation.
\end{abstract}

1991 Mathematics Subject Classification. 34E15, 58F14, 93D15.

Key words and phrases. Hopf bifurcation, nonlinear control theory, singular perturbations, dynamic bifurcations, codimension four unfolding, Liénard equation. 


\section{Introduction}

The main motivation for this paper is the problem of ageing of dynamical systems. Assume that the behaviour of a device can be described by an $n$-dimensional ordinary differential equation $\dot{x}=f(x)$. The system is designed in such a way that $x^{\star}$ is an asymptotically stable equilibrium point, which corresponds to the desired behaviour. Slow changes of the system's characteristics due to the ageing process can be modelled by a slowly timedependent equation

$$
\frac{\mathrm{d} x}{\mathrm{~d} t}=f(x, \varepsilon t), \quad 0<\varepsilon \ll 1,
$$

where $f(x, 0)$ describes the dynamics of the brand new device.

Equation (1) being a nonautonomous differential equation which is difficult to solve, one is tempted to consider instead the 1-parameter family of autonomous systems

$$
\frac{\mathrm{d} x}{\mathrm{~d} t}=f(x, \lambda), \quad \lambda=\text { constant. }
$$

One hopes that if the "quasistatic approximation" (2) has a family of attractors depending smoothly on $\lambda$, then solutions of (1) should be close, at any given time $t$, to the attractor of (2) with $\lambda=\varepsilon t$.

This is at least partially justified by the following result [PR, $\mathrm{VBK}, \mathrm{Fe}]$ : if $x^{\star}(\lambda)$ is a family of asymptotically stable equilibria of (2), then any solution of (1) starting in a sufficiently small neighbourhood of $x^{\star}(0)$ will, after a short transient, track the curve $x^{\star}(\varepsilon t)$ at a distance of order $\varepsilon$. For the ageing device, this implies that we need not worry as long as the "nominal" equilibrium $x^{\star}(\lambda)$ remains asymptotically stable.

This naturally raises the question of what happens if the equilibrium $x^{\star}(\lambda)$ undergoes a bifurcation at $\lambda=\lambda_{0}$. Such problems are usually referred to as dynamic bifurcations Ben. Bifurcations with a single zero eigenvalue have been studied in some detail. It turns out that saddle-node bifurcations will result in a sudden jump of the solution [Hab], which may have catastrophic consequences for the device. Transcritical and pitchfork bifurcations generically result in a smoother behaviour, where the trajectory follows one of the stable equilibria created in the bifurcation [LS]. This feature might be used to detect the loss of stability of the nominal equilibrium, in order to switch off the device before any harm is done.

The case of a Hopf bifurcation has been analysed more recently [Sh, Ne1, Ne2]. The surprising phenomenon is that instead of directly tracking the limit cycle created in the bifurcation, the trajectory remains close to the unstable equilibrium for some time, before jumping to the periodic orbit. Instead of oscillations with a continuously increasing amplitude, one thus observes the sudden appearance, after some delay, of large amplitude oscillations (Fig. 17a). The bifurcation delay is stable with respect to smooth deterministic perturbations. For the ageing device, this phenomenon implies that the loss of stability cannot be detected soon enough to avoid catastrophic oscillations.

To avoid such problems, one may try to control the system. A simple affine control of the quasistatic system (2) would be

$$
\frac{\mathrm{d} x}{\mathrm{~d} t}=f(x, \lambda)+b u
$$

where $b$ is a given vector in $\mathbb{R}^{n}$ and $u$ is a scalar function. Two cases have been studied: 
1. Open loop control: $u(t)$ is a function of time taking values in some compact interval $U$. The analysis of (3) for all possible functions of this type leads to the notion of control sets, which tend to form around invariant sets. The $\lambda$-dependence of control sets near bifurcation points has been studied in [CK, CHK, HS].

2. Feedback control: $u(x(t))$ is a function of the state of the system. The bifurcation is "stabilized" by choosing the function $u(x)$ in such a way that the nominal equilibrium of (3) is stable when $\lambda=\lambda_{0}$. For topological reasons, this makes the bifurcation supercritical, and should avoid exploding trajectories at least for $\lambda$ slightly larger than $\lambda_{0} \mathrm{Ab}, \mathrm{AB}, \mathrm{MS}$.

The sometimes surprising behaviour of dynamic bifurcations shows that we should consider the slowly time-dependent version of (3) as well. Here we will limit ourselves to feedback controlled systems of the form

$$
\frac{\mathrm{d} x}{\mathrm{~d} t}=f(x, \varepsilon t)+b u(x, \varepsilon t)
$$

in the specific case where $f$ undergoes a Hopf bifurcation. Since we wish to analyse (田) on the time scale $\varepsilon^{-1}$, we introduce the slow time $\tau=\varepsilon t$ and rewrite (4) as the singular perturbation problem

$$
\varepsilon \frac{\mathrm{d} x}{\mathrm{~d} \tau}=f(x, \tau)+b u(x, \tau)
$$

Our aim is to design a feedback control $u$ in such a way that the bifurcation delay, and hence the sudden appearance of large amplitude oscillations, are suppressed.

A feedback affecting only nonlinear terms in $x-x^{\star}$ will have no effect on the delay. We thus have to modify the linearization $A$ of $f$ at the bifurcation point. Shifting the real part of the eigenvalues of $A$ will merely postpone the problem to some later time. The only solution is thus to shift the imaginary part of the eigenvalues in order to produce a double zero eigenvalue.

Our strategy will thus be the following. First we design a feedback in such a way that when the parameter $\lambda$ is varied through the bifurcation value $\lambda_{0}$, new stable equilibrium branches are created at a distance of order $\left(\lambda-\lambda_{0}\right)^{1 / 2}$ of the nominal branch $x^{\star}(\lambda)$. The resulting vector field turns out to be a codimension four unfolding studied in KKR. We then show that the solutions of the corresponding system (5) actually track one of these branches, a feature which can be used to detect the bifurcation point.

To analyse the time-dependent system, we extend certain methods of [B1] (see [B2] for a summary and [BK for applications). However, this paper is written in a largely self-contained way. It is organized as follows: the main result is stated in Section 2; in Section 3 we recall Neishtadt's result on bifurcation delay [Ne1], which will guide us in the construction of the feedback in Section 4 . Section 5 is devoted to the proof of the main result for the singularly perturbed equation (5). Finally, in Section 6, we give a few remarks on what happens when the control is slightly imperfect, and the requirements of the theorem are no longer met.

Acknowledgments: It is a pleasure to thank Klaus Schneider for welcoming me at the Weierstraß Institute and for inspiring discussions on this interesting topic. I thank Dima Turaev for pointing out useful references on high codimension bifurcations. This work is supported by the Nonlinear Control Network of the European Community, Grant ERB FMRXCT-970137. 


\section{The Problem and Main Result}

We consider the feedback controlled dynamical system

$$
\frac{\mathrm{d} x}{\mathrm{~d} t}=f(x, \lambda)+b u(x, \lambda), \quad x \in \mathbb{R}^{n}, \lambda \in \mathbb{R}
$$

where:

- The uncontrolled vector field $f(x, \lambda)$ undergoes a Poincaré-Andronov-Hopf bifurcation at the origin.

- The vector $b \in \mathbb{R}^{n}$ is imposed and fixed. It describes the direction in which the system can be steered.

- The scalar feedback control $u(x, \lambda) \in \mathbb{R}$ is a function to be determined in such a way that the solution behaves "smoothly" when $\lambda$ is slowly varied. Its dependence on $x$ and $\lambda$ should be as simple as possible (e.g. polynomial).

More precisely, we will assume that the uncontrolled vector field satisfies the following hypotheses:

(H1) Domain and smoothness: The function $f(x, \lambda): \mathcal{D} \times I \rightarrow \mathbb{R}^{n}$ is analytic in a neighbourhood $\mathcal{D}$ of the origin in $\mathbb{R}^{n}$ and an interval $I$ containing 0 .

(H2) Hopf bifurcation: There exists a curve $x^{\star}(\lambda): I \rightarrow \mathbb{R}^{n}$ with $x^{\star}(0)=0$ and $f\left(x^{\star}(\lambda), \lambda\right)=0$. The Jacobian matrix $A(\lambda)=\partial_{x} f\left(x^{\star}(\lambda), \lambda\right) \in \mathbb{R}^{n \times n}$ admits two eigenvalues $a(\lambda) \pm \mathrm{i} \omega(\lambda)$, where $a(0)=0, a^{\prime}(0)>0$ and $\omega(0)=\omega_{0} \neq 0$. All other eigenvalues of $A(\lambda)$ have a strictly negative real part.

These hypotheses imply in particular that there is a set of coordinates $x=(y, z)$, with $y \in \mathbb{R}^{m}(m=n-2)$ and $z=(\xi, \eta) \in \mathbb{R}^{2}$, such that

$$
f(x, 0)=\left(\begin{array}{c}
A_{-} y+g_{-}(y, z) \\
A_{0} z+g_{0}(y, z)
\end{array}\right), \quad A_{0}=\left(\begin{array}{cc}
0 & \omega_{0} \\
-\omega_{0} & 0
\end{array}\right),
$$

where all eigenvalues of $A_{-} \in \mathbb{R}^{m \times m}$ have negative real part and $g_{-}$and $g_{0}$ are of second order in $y$ and $z$. In these coordinates, we write $b=\left(\begin{array}{c}b_{-} \\ b_{0}\end{array}\right)$.

(H3) Controllability: $b_{0} \neq 0$.

Since $A_{0}$ is rotation invariant, we may assume that $b_{0}=\left(\begin{array}{l}0 \\ 1\end{array}\right)$.

The next hypothesis is more technical and its meaning will become clear in Section 4 . It is, however, generically satisfied.

(H4) Nondegeneracy: Define the matrix $T=-\left(A_{-}^{-1} b_{-}, A_{-}^{-2} b_{-}\right) \in \mathbb{R}^{m \times 2}$. Let $g_{\xi}$ denote the first component of $g_{0}$, and $h(z)=g_{\xi}(T z, z)$. Then either $\partial_{\xi \xi} h(0) \neq 0$ or $\partial_{\xi \xi \xi} h(0)<0$.

Finally, we require the following property of the equilibrium branch $x^{\star}(\lambda)$ :

(H5) Velocity of equilibrium: The $\eta$-component of $\left.\mathrm{d}_{\lambda} x^{\star}(\lambda)\right|_{\lambda=0}$ is different from zero.

The main result of this paper is the following. 

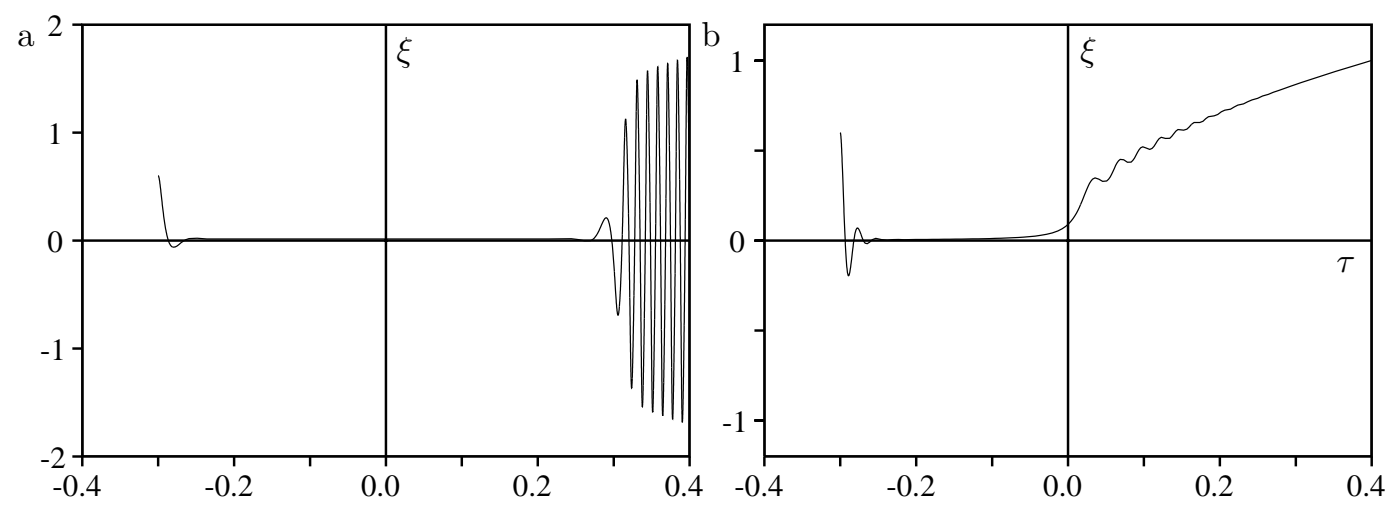

Figure 1. (a) The slow passage through a Hopf bifurcation (occurring at $\tau=0$ ) leads to the delayed appearance of oscillations. (b) The control we construct suppresses this delay, and causes the orbit to track a curve lying at a distance of order $\tau^{1 / 2}$ from the nominal equilibrium (represented by the axis $\xi=0$ ). These plots actually show a solution $\xi(\tau)$ of equation (11) with $a(\tau)=\tau, \gamma(\tau)=\delta(\tau)=0, R(\xi, \eta, \tau, \varepsilon)=1, \varepsilon=0.003$ and (a) $\mu(\tau)=-0.2$, (b) $\mu(\tau)=2.5 \tau$. The eigenvalues of the linearization around the origin are $\tau \pm \mu^{1 / 2}$.

Theorem 2.1. Assume that the hypotheses (H1)-(H5) hold. There exist

- strictly positive constants $T, M$ and $\kappa$,

- a neighbourhood $\mathcal{N} \subset \mathcal{D}$ of the origin in $\mathbb{R}^{n}$,

- a smooth feedback control $u(x, \lambda): \mathcal{N} \times[-T, T] \rightarrow \mathbb{R}$ with $u\left(x^{\star}(\lambda), \lambda\right)=0$,

- and a curve $x_{+}(\lambda):[0, T] \rightarrow \mathbb{R}^{n}$ with $\lim _{\lambda \rightarrow 0+}\left\|x_{+}(\lambda)-x^{\star}(\lambda)\right\| / \sqrt{\lambda}=K \neq 0$

with the following property. For every $\tau_{0} \in[-T, 0)$, there exist strictly positive constants $c_{1}$ and $\varepsilon_{0}$ such that, if $0<\varepsilon<\varepsilon_{0}$, any solution of the equation

$$
\varepsilon \frac{\mathrm{d} x}{\mathrm{~d} \tau}=f(x, \tau)+b u(x, \tau)
$$

with initial condition $x\left(\tau_{0}\right) \in \mathcal{N}$ exists on the interval $\left[\tau_{0}, T\right]$ and satisfies the following bounds:

$$
\begin{array}{rlrl}
\left\|x(\tau)-x^{\star}(\tau)\right\| & \leqslant M \frac{\varepsilon}{|\tau|}, & \tau_{0}+c_{1} \varepsilon|\ln \varepsilon| \leqslant \tau \leqslant-\varepsilon^{2 / 3}, \\
\left\|x(\tau)-x^{\star}(\tau)\right\| \leqslant M \varepsilon^{1 / 3}, & -\varepsilon^{2 / 3} \leqslant \tau \leqslant \varepsilon^{2 / 3}, \\
\left\|x(\tau)-x_{+}(\tau)\right\| \leqslant M\left(\frac{\varepsilon}{\tau}+\frac{\varepsilon^{1 / 2}}{\tau^{1 / 4}} \mathrm{e}^{-\kappa \tau^{2} / \varepsilon}\right), & \varepsilon^{2 / 3} \leqslant \tau \leqslant T .
\end{array}
$$

This theorem shows that solutions of (8) will first track the nominal equilibrium curve $x^{\star}(\tau)$ for negative $\tau$, and then track a new equilibrium curve $x_{+}(\tau)$, situated at a distance of order $\sqrt{\tau}$ from $x^{\star}(\tau)$, for positive $\tau$ (Fig. 1 $\mathrm{b}$ ). This result looks similar to the stability exchange for pitchfork bifurcations in [LS]. It is, however, more difficult to obtain because the specific nature of the bifurcation with double zero eigenvalue makes the problem intrinsically two-dimensional. In particular, solutions tend to rotate around the equilibrium branches which are foci near the bifurcation point.

The proof is divided into two main steps. In the first one, described in Section 4, we consider the autonomous system (6). We construct a feedback $u(x, \lambda)$ in such a way that after some changes of variables, including a center manifold reduction and a transformation 
to normal form, the dynamics is governed by the two-dimensional effective equation

$$
\begin{aligned}
& \frac{\mathrm{d} \xi}{\mathrm{d} t}=\eta \\
& \frac{\mathrm{d} \eta}{\mathrm{d} t}=\mu(\lambda) \xi+2 a(\lambda) \eta+\gamma(\lambda) \xi^{2}+\delta(\lambda) \xi \eta-\xi^{3}-\xi^{2} \eta+\mathcal{O}(4)
\end{aligned}
$$

where $\mu(0)=\gamma(0)=\delta(0)=0$ and $\mathcal{O}(4)=\mathcal{O}\left(\|(\xi, \eta)\|^{4}\right)$. The function $\mu(\lambda)$ can be controlled to some extent by the choice of $u$. This so-called cubic Liénard equation happens to be a codimension- 4 unfolding of the vector field $\left(\eta,-\xi^{3}-\xi^{2} \eta\right)$ which has been studied in detail, see [KKR and references therein (the authors in [KKR actually use an equivalent unfolding obtained by the transformation $\left.\xi \mapsto \xi+\frac{1}{3} \gamma\right)$.

The second step is to show that with this particular feedback, the slowly time-dependent system (8) can be reduced to

$$
\begin{aligned}
& \varepsilon \frac{\mathrm{d} \xi}{\mathrm{d} \tau}=\eta \\
& \varepsilon \frac{\mathrm{d} \eta}{\mathrm{d} \tau}=\mu(\tau) \xi+2 a(\tau) \eta+\gamma(\tau) \xi^{2}+\delta(\tau) \xi \eta-\xi^{3}-\xi^{2} \eta+\mathcal{O}(4)+\varepsilon R(\xi, \eta, \tau, \varepsilon) .
\end{aligned}
$$

The function $R(0,0, \tau, 0)$ is related to the drift $\mathrm{d} x^{\star}(\tau) / \mathrm{d} \tau$ of the equilibrium branch, and Hypothesis (H5) implies that $R(0,0,0,0) \neq 0$. In Section 5 we prove the following result.

Theorem 2.2. Assume that $\mu^{\prime}(0)>0$. There exist positive constants $d, T, M, \kappa$ and $a$ neighbourhood $\mathcal{M}$ of the origin in $\mathbb{R}^{2}$ with the following property. For every $\tau_{0} \in[-T, 0)$, there is a constant $c_{1}>0$ such that for sufficiently small $\varepsilon$, any solution of (11) with initial condition $(\xi, \eta)\left(\tau_{0}\right) \in \mathcal{M}$ satisfies

$$
\begin{array}{lll}
|\xi(\tau)| \leqslant M \frac{\varepsilon}{|\tau|}, & |\eta(\tau)| \leqslant M \frac{\varepsilon}{|\tau|^{1 / 2}}, & \text { for } \tau_{1}(\varepsilon) \leqslant \tau \leqslant-\left(\frac{\varepsilon}{d}\right)^{2 / 3}, \\
|\xi(\tau)| \leqslant M \varepsilon^{1 / 3}, & |\eta(\tau)| \leqslant M \varepsilon^{2 / 3}, & \text { for }-\left(\frac{\varepsilon}{d}\right)^{2 / 3} \leqslant \tau \leqslant\left(\frac{\varepsilon}{d}\right)^{2 / 3},
\end{array}
$$

where $\tau_{1}(\varepsilon)=\tau_{0}+c_{1} \varepsilon|\ln \varepsilon|$. If, moreover, the relations

$$
\frac{a^{\prime}(0)}{\mu^{\prime}(0)}<\frac{1}{2}, \quad R(0,0,0,0) \neq 0
$$

hold, then for $(\varepsilon / d)^{2 / 3} \leqslant \tau \leqslant T$ we have

$$
\begin{aligned}
\left|\xi(\tau)-\xi_{+}(\tau)\right| & \leqslant M\left[\frac{\varepsilon}{\tau}+\frac{\varepsilon^{1 / 2}}{\tau^{1 / 4}} \mathrm{e}^{-\kappa \tau^{2} / \varepsilon}\right] \\
|\eta(\tau)| & \leqslant M\left[\frac{\varepsilon}{\tau^{1 / 2}}+\varepsilon^{1 / 2} \tau^{1 / 4} \mathrm{e}^{-\kappa \tau^{2} / \varepsilon}\right]
\end{aligned}
$$

where

$$
\xi_{+}(\tau)= \begin{cases}\sqrt{\mu}+\mathcal{O}(\tau), & \text { if } R(0,0,0,0)>0, \\ -\sqrt{\mu}+\mathcal{O}(\tau), & \text { if } R(0,0,0,0)<0\end{cases}
$$

are equilibria of (10), i.e., the right-hand side of (10) vanishes when $\xi=\xi_{+}$and $\eta=0$. 


\section{The Uncontrolled Problem}

We state a version of Neishtadt's result on bifurcation delay for the uncontrolled problem

$$
\varepsilon \frac{\mathrm{d} x}{\mathrm{~d} \tau}=f(x, \tau)
$$

The results in Ne1, Ne2 apply in fact to a more general slow-fast system. We are interested in the computation of the delay time. To this end, we define the function

$$
\Psi(\tau)=\int_{0}^{\tau} \rho(s) \mathrm{d} s, \quad \rho(s)=a(s)+\mathrm{i} \omega(s) .
$$

In some interval $(-T, 0)$ in which $a\left(\tau_{0}\right)<0$ we can define the map

$$
\Pi\left(\tau_{0}\right)=\sup _{\tau>\tau_{0}}\left\{\tau \mid \operatorname{Re} \psi(s)<\operatorname{Re} \psi\left(\tau_{0}\right), \tau_{0}<s<\tau\right\},
$$

giving the first time at which $\operatorname{Re} \psi(\tau)$ becomes equal to $\operatorname{Re} \psi\left(\tau_{0}\right)$ again. Observe that $\Pi\left(\tau_{0}\right)>0, \lim _{\tau_{0} \rightarrow 0} \Pi(\tau)=0$ and $\lim _{\tau_{0} \rightarrow 0} \Pi^{\prime}\left(\tau_{0}\right)=-1$.

Theorem 3.1. Assume that $f(x, \lambda)$ satisfies Hypotheses (H1) and (H2) for all $(x, \lambda)$ in some complex neighbourhood of $\mathcal{D} \times I$. Let $\tau_{0}<0$ be such that $a(\tau)<0$ for $\tau_{0} \leqslant \tau<0$. There exist a neighbourhood $\mathcal{N}$ of $x^{\star}\left(\tau_{0}\right)$, a constant $M>0$, a buffer time $\tau_{+}>0$ and a continuous function $\delta(\varepsilon)$ going to 0 as $\varepsilon \rightarrow 0$, such that any solution of (17) with initial condition $x\left(\tau_{0}\right) \in \mathcal{N}$ satisfies

$$
\left\|x(\tau)-x^{\star}(\tau)\right\| \leqslant M \varepsilon \quad \text { for } \tau_{0}+\delta(\varepsilon) \leqslant \tau \leqslant \hat{\tau}-\delta(\varepsilon),
$$

where the bifurcation delay time $\hat{\tau}$ is given by

$$
\hat{\tau}=\min \left\{\Pi\left(\tau_{0}\right), \tau_{+}\right\} .
$$

The quantity $\hat{\tau}$ gives in fact a lower bound on the bifurcation delay. Under more strict assumptions, it also gives an upper bound in the limit $\varepsilon \rightarrow 0$, see Ne2 and the article by F. and M. Diener in [Ben].

The important fact for us is that $\hat{\tau}$ is the minimum of two quantities. The first one, $\Pi\left(\tau_{0}\right)$, depends on the initial condition and thus cannot be used to influence the delay. We thus have to modify the buffer time $\tau_{+}$, which is determined in the following way. The function $\Psi(\tau)$ and the solutions of (17) can be continued to a complex neighbourhood of $\tau=0$. For sufficiently small $\left|\tau_{0}\right|$, the real times $\tau_{0}$ and $\Pi\left(\tau_{0}\right)$ can be connected by a path $\Gamma\left(\tau_{0}\right)$ (lying in the upper half plane if $\omega_{0}<0$ ), along which $\operatorname{Re} \Psi(\tau)$ is constant. One defines a negative buffer time $\tau_{-}$as the smallest real time such that $\Gamma\left(\tau_{-}\right)$exists and has certain properties stated in Ne2]. The positive buffer time is given by

$$
\tau_{+}=\sup _{\tau_{-}<\tau<0} \Pi(\tau) .
$$

The existence of this buffer time is a rather subtle, nonperturbative effect, which cannot be understood by naive perturbation theory. The proof uses an integration of (17) along a path $\Gamma(\tau)$.

Example 3.2. If $a(\tau)=\tau$ and $\omega(\tau)=-\omega_{0}$ i, then the level lines of

$$
\operatorname{Re} \Psi(\tau)=\frac{1}{2}\left[(\operatorname{Re} \tau)^{2}-\left(\operatorname{Im} \tau-\omega_{0}\right)^{2}+\omega_{0}^{2}\right]
$$

are hyperbolas centered at $\tau=\mathrm{i} \omega_{0}$. Thus we have $\Pi\left(\tau_{0}\right)=-\tau_{0}$ and the buffer times are $\tau_{ \pm}= \pm \omega_{0}$. The delay may be suppressed by choosing a control in such a way that $\omega_{0}=0$. 


\section{Design of the Feedback Control}

We start by analysing (6) for $\lambda=0$. Coordinates are chosen as in (7), where we can scale time in such a way that $\omega_{0}=1$. Our feedback control will be constructed to satisfy two requirements:

1. The properties of the bifurcation delay time show that we should move the imaginary part of the eigenvalues $\pm \omega(0)$ towards the origin, in order to shift the buffer time to the bifurcation time.

2. This will produce a bifurcation with double zero eigenvalue. In analogy with works on stabilization of bifurcations [As, MS, the equilibrium should be stable at the bifurcation point, in order to avoid escaping trajectories.

We claim that an appropriate feedback control has the form

$$
u(x, 0)=(1-\nu) \xi+v(z) .
$$

[We recall that $x=(y, z) \in \mathbb{R}^{m} \times \mathbb{R}^{2}$ and $z=(\xi, \eta)$.] The parameter $\nu$ will be ultimately set to 0 , we introduce it in order to keep track of the effect of an imperfect control. Its aim is to shift the eigenvalues $\pm \mathrm{i} \sqrt{\nu}$ of the linearization to the origin. The function $v(z)$ is a nonlinear term which should assure that the origin of (6) is stable.

\subsection{Center Manifold Reduction at $\lambda=0$}

With the feedback control (24), equation (6) takes the form

$$
\begin{aligned}
& \dot{y}=A_{-} y+\hat{g}_{-}(y, z)+B_{-} z \\
& \dot{z}=\hat{A}_{0} z+\hat{g}_{0}(y, z),
\end{aligned}
$$

where $\hat{g}_{-}=g_{-}(x)+b v(z), \hat{g}_{0}=g_{0}(x)+b v(z), B_{-}=(1-\nu)\left(b_{-}, 0\right) \in \mathbb{R}^{m \times 2}$ and the marginally stable part of the linearization is given by the matrix $\hat{A}_{0}(\nu)=\left(\begin{array}{cc}0 & 1 \\ -\nu & 0\end{array}\right)$.

Proposition 4.1. Equation (25) admits an invariant manifold on which the dynamics is governed by the equation

$$
\dot{z}=\hat{A}_{0} z+G(z)
$$

where $G(z)=\mathcal{O}\left(\|z\|^{2}\right)$ is given to third order by equation (31) below.

Proof: The existence of the manifold follows quite directly from the Center Manifold Theorem [Ca]. We would now like to compute $G$. First, we introduce the matrix $T \in \mathbb{R}^{m \times 2}$ satisfying $A_{-} T-T \hat{A}_{0}=-B_{-}$, which exists because $A_{-}$and $\hat{A}_{0}$ have no eigenvalues in common $\mathrm{Kr}$, Wa. In fact, it is given by

$$
T=\left(t_{1}, t_{2}\right), \quad t_{1}=A_{-} t_{2}, \quad t_{2}=(\nu-1)\left(A_{-}^{2}+\nu \mathbb{1}\right)^{-1} b_{-} .
$$

The change of variables $y=y_{1}+T z$ yields

$$
\begin{aligned}
\dot{y}_{1} & =A_{-} y_{1}+\tilde{g}_{-}\left(y_{1}, z\right), & \tilde{g}_{-}\left(y_{1}, z\right) & =\hat{g}_{-}\left(y_{1}+T z, z\right)-T \hat{g}_{0}\left(y_{1}+T z, z\right), \\
\dot{z} & =\hat{A}_{0} z+\tilde{g}_{0}\left(y_{1}, z\right), & \tilde{g}_{0}\left(y_{1}, z\right) & =\hat{g}_{0}\left(y_{1}+T z, z\right) .
\end{aligned}
$$


This system admits a center manifold locally described by $y_{1}=h(z)$, where $h(z)$ satisfies the partial differential equation

$$
A_{-} h(z)+\tilde{g}_{-}(h(z), z)=\partial_{z} h(z)\left[\hat{A}_{0} z+\tilde{g}_{0}(h(z), z)\right] .
$$

For a vector field $F(x): \mathbb{R}^{n} \rightarrow \mathbb{R}^{m}$, we denote by $F^{(k)}(x)$ the terms of order $k$ of its Taylor expansion around 0. We know [Ca] that $h(z)=h^{(2)}(z)+\mathcal{O}\left(\|z\|^{3}\right)$, where

$$
\partial_{z} h^{(2)}(z) \hat{A}_{0} z-A_{-} h^{(2)}(z)=\tilde{g}_{-}^{(2)}(0, z) .
$$

The motion on the center manifold is thus given by (26), where

$$
\begin{aligned}
G(z) & =\hat{g}_{0}(h(z)+T z, z) \\
& =G^{(2)}(z)+G^{(3)}(z)+\mathcal{O}\left(\|z\|^{4}\right), \\
G^{(2)}(z) & =\hat{g}_{0}^{(2)}(T z, z) \\
G^{(3)}(z) & =\hat{g}_{0}^{(3)}(T z, z)+\partial_{y} \hat{g}_{0}^{(2)}(T z, z) h^{(2)}(z) .
\end{aligned}
$$

To compute $G^{(3)}(z)$, we need to solve equation (30) for $h^{(2)}$. In fact, we will only need to know that $h^{(2)}(z)=-A_{-}^{-1} \tilde{g}^{1} \xi^{2}+\mathcal{O}(\eta)$, where $\tilde{g}^{1}$ is the coefficient of $\xi^{2}$ in $\tilde{g}_{-}^{(2)}(0, z)$.

\subsection{Stability at $\lambda=0$}

We intend to construct the nonlinear part of the feedback control of the form

$$
v(z)=v_{1} \xi^{2}+v_{2} \xi \eta+v_{3} \eta^{2}+v_{4} \xi^{3}
$$

with appropriate coefficients $v_{i}$. They will be determined by the following result of normal form theory:

Lemma 4.2. Consider the system

$$
\begin{aligned}
& \dot{\xi}=\eta+c_{1} \xi^{2}+c_{2} \xi \eta+c_{3} \eta^{2}+c_{4} \xi^{3}+\mathcal{O}\left(\eta\|z\|^{2},\|z\|^{4}\right) \\
& \dot{\eta}=\quad d_{1} \xi^{2}+d_{2} \xi \eta+d_{3} \eta^{2}+d_{4} \xi^{3}+\mathcal{O}\left(\eta\|z\|^{2},\|z\|^{4}\right) .
\end{aligned}
$$

Let $\alpha=c_{1} d_{3}+c_{4}$ and $\beta=2 c_{1}^{2}+d_{4}$. Then

- If $d_{1}=d_{2}+2 c_{1}=0$ and $\alpha, \beta<0$, the origin is asymptotically stable.

- Conversely, if the origin is stable, then $d_{1}=d_{2}+2 c_{1}=0$ and $\alpha, \beta \leqslant 0$.

PROOF: The normal form of the 3-jet of (33) can be written as

$$
\begin{aligned}
& \dot{\xi}=\eta \\
& \dot{\eta}=\gamma \xi \eta+\delta \xi^{2}+\alpha \xi^{2} \eta+\beta \xi^{3},
\end{aligned}
$$

with $\gamma=d_{2}+2 c_{1}$ and $\delta=d_{1}$. The assertion has been proved in [Ta], see also [GH]. In fact, the origin is an unstable Bogdanov-Takens point if $\gamma \neq 0$ or if $\delta \neq 0$. If $\gamma=\delta=0$, it is asymptotically stable if $\alpha$ and $\beta$ are both negative, and unstable if one of them is positive.

We would like to choose the coefficients of $v(z)$ in such a way that the vector field (26) satisfies Lemma 4.2. The next lemma shows that this is generically possible. 
Lemma 4.3. Assume that the $\xi$-component of $g_{0}(T z, z)$ has the form $c_{1} \xi^{2}+c_{4} \xi^{3}+\mathcal{O}\left(\eta, \xi^{4}\right)$. Then, if either $c_{1} \neq 0$ or $c_{4}<0$, one can find a function $v(z)$ of the form (32) such that the origin in (26) is asymptotically stable.

ProOF: Let us consider

$$
G^{(2)}(z)=\hat{g}_{0}^{(2)}(T z, z)=\left(\begin{array}{c}
c_{1} \xi^{2}+c_{2} \xi \eta+c_{3} \eta^{2} \\
\left(d_{1}+v_{1}\right) \xi^{2}+\left(d_{2}+v_{2}\right) \xi \eta+\left(d_{3}+v_{3}\right) \eta^{2}
\end{array}\right) .
$$

The first two conditions of Lemma 4.2 are satisfied if we choose $v_{1}=-d_{1}$ and $v_{2}=$ $-\left(d_{2}+2 c_{1}\right)$. To satisfy the third one, we have to choose $v_{3}$ in such a way that $\alpha=$ $c_{1}\left(d_{3}+v_{3}\right)+c_{4}<0$, which is possible under our assumptions. The last condition looks more difficult to satisfy, but in fact, we have $\beta=v_{4}+$ constant, where the constant depends only on previously fixed quantities, so that $\beta$ can always be made negative.

The requirement on $g_{0}(T z, z)$ is nothing but Hypothesis (H4). Note that the coefficients $v_{3}$ and $v_{4}$ only have to satisfy inequalities, while $v_{1}$ and $v_{2}$ must have a specific value. We will discuss in Section 6 what happens when these coefficients are not exactly equal to the prescribed value.

\subsection{Choice of $u(x, \lambda)$}

We consider now equation (6) for general, fixed values of $\lambda$. If $x^{\star}(\lambda)$ is the equilibrium branch of $f$, an affine transformation $x=x^{\star}(\lambda)+S(\lambda)\left(\begin{array}{c}y \\ z\end{array}\right)$ yields the system

$$
\begin{aligned}
& \dot{y}=A_{-}(\lambda) y+g_{-}(y, z, \lambda)+b_{-}(\lambda) \tilde{u}(y, z, \lambda) \\
& \dot{z}=A_{0}(\lambda) z+g_{0}(y, z, \lambda)+b_{0}(\lambda) \tilde{u}(y, z, \lambda),
\end{aligned}
$$

where $A_{-}(\lambda)$ has eigenvalues with negative real part for sufficiently small $\lambda$, and

$$
A_{0}(\lambda)=\left(\begin{array}{cc}
a(\lambda) & \omega(\lambda) \\
-\omega(\lambda) & a(\lambda)
\end{array}\right)
$$

In fact, since $A_{0}$ is rotation invariant, we may assume that $b_{0}(\lambda) \equiv\left(\begin{array}{l}0 \\ 1\end{array}\right)$. The terms $g_{-}$ and $g_{0}$ are nonlinear.

We now choose the feedback control $u(x, \lambda)$ in such a way that

$$
\tilde{u}(y, z, \lambda)=(1+C \lambda)[(1-\nu) \xi+v(z)]
$$

where $C$ is some constant to be determined, and $v(z)$ has been constructed in the previous section. In this way, the linear part of $\dot{z}$ becomes

$$
\hat{A}_{0}(\lambda)=\left(\begin{array}{cc}
a(\lambda) & \omega(\lambda) \\
-\omega(\lambda)+(1-\nu)(1+C \lambda) & a(\lambda)
\end{array}\right) .
$$

This matrix can be further simplified by a shearing transformation:

$$
z \mapsto\left(\begin{array}{cc}
\omega^{1 / 2} & 0 \\
0 & \omega^{-1 / 2}
\end{array}\right) z \quad \Rightarrow \quad \hat{A}_{0}(\lambda) \mapsto B(\lambda)=\left(\begin{array}{cc}
a(\lambda) & 1 \\
\mu(\lambda) & a(\lambda)
\end{array}\right),
$$

where

$$
\mu(\lambda)=-\omega^{2}+(1-\nu)(1+C \lambda) \omega=\left(C-\omega^{\prime}(0)\right) \lambda-\nu+\mathcal{O}\left(\lambda^{2}+\nu^{2}\right) .
$$




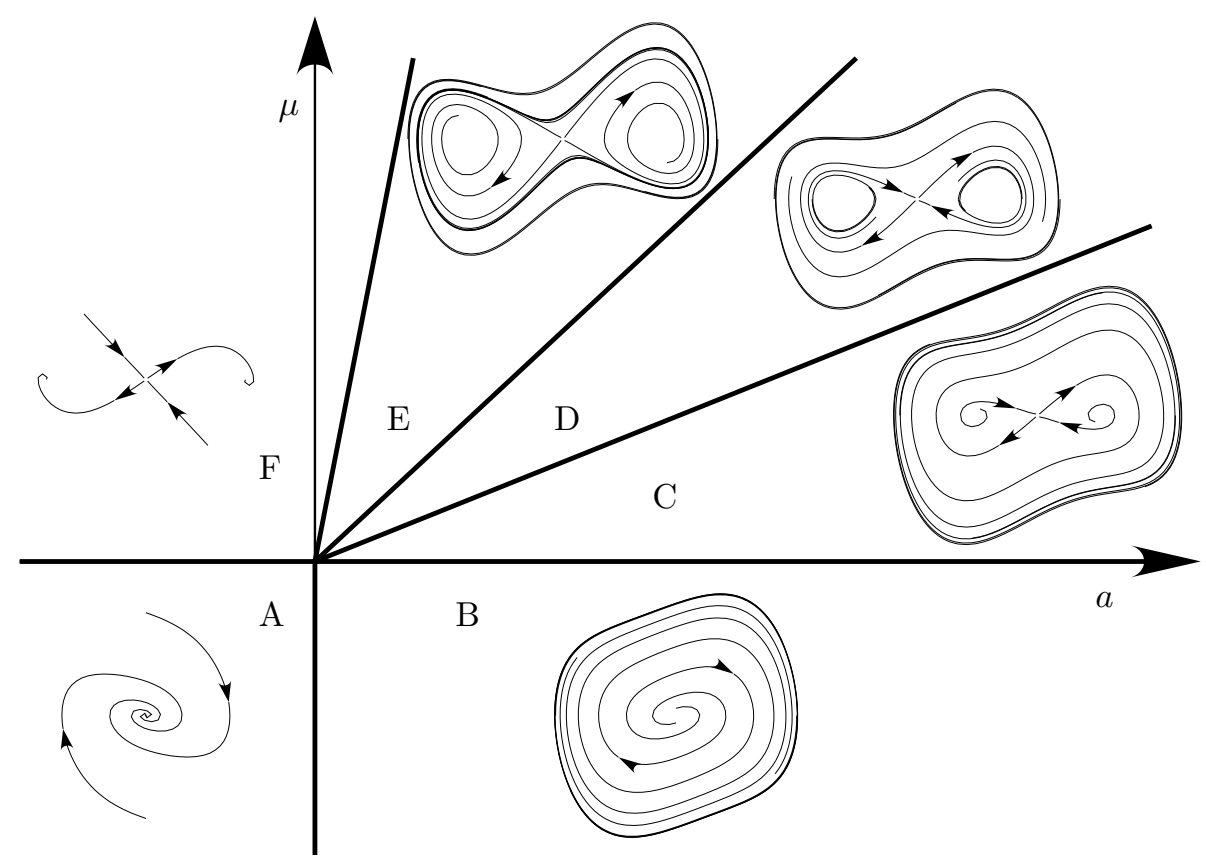

FIgURE 2. Schematic bifurcation diagram of equation (43a) in the plane $\hat{\gamma}=\hat{\delta}=0$. The line A-B is the original Hopf bifurcation. By moving the eigenvalues' imaginary parts to 0 , we change the function $\mu(\lambda)$ in such a way that $\mu(0)=0$. This produces new bifurcation lines. The transition A-F is a supercritical saddle-node bifurcation, the transition C-B a subcritical one. D-C is a subcritical Hopf bifurcation, D-E a homoclinic bifurcation and E-F a saddle-node bifurcation of periodic orbits.

In fact, any of the pairs $(\lambda, \nu),(\lambda, \mu)$ or $(a, \mu)$ can be considered as independent parameters used to produce the bifurcation with double zero eigenvalue. We will henceforth set $\nu=0$ and consider $\left.(a(\lambda), \mu(\lambda))=\left(C-\omega^{\prime}(0)\right) \lambda+\mathcal{O}\left(\lambda^{2}\right)\right)$ as a path going through the origin of the two-dimensional parameter space.

The system (36) admits a center manifold described locally by its parametric equation $z=h(y, \lambda)$. On this manifold, the dynamics is governed by the equation

$$
\dot{z}=B(\lambda) z+G(z, \lambda) .
$$

It will not be necessary to compute $G(z, \lambda)$. We only need to know that $G(z, 0)$ has been computed in Proposition 4.1 and satisfies the requirements of Lemma 4.2 .

\subsection{Normal Forms}

The nonlinear term $G(z, \lambda)$ can be simplified by eliminating all terms which are not resonant at $\lambda=0$. A convenient basis of resonant terms up to order 3 is given by $\left\{\left(0, \xi^{2}\right),(0, \xi \eta),\left(0, \xi^{3}\right),\left(0, \xi^{2} \eta\right)\right\}$.

Proposition 4.4. The 3-jet of (42) can be written in either of the following forms:

$$
\begin{aligned}
& \dot{\xi}=a \xi+\eta \\
& \dot{\eta}=\mu \xi+a \eta+\hat{\gamma} \xi^{2}+\hat{\delta} \xi \eta-\xi^{3}-\xi^{2} \eta,
\end{aligned}
$$




$$
\begin{aligned}
& \dot{\xi}=\eta \\
& \dot{\eta}=\alpha \xi+\beta \eta+\gamma \xi^{2}+\delta \xi \eta-\xi^{3}-\xi^{2} \eta \\
& \dot{\xi}=\eta \\
& \dot{\eta}=\mu_{1}+\mu_{2} \xi+\mu_{3} \eta+\mu_{4} \xi \eta-\xi^{3}-\xi^{2} \eta
\end{aligned}
$$

Each set of parameters $(a, \mu, \hat{\gamma}, \hat{\delta}),(\alpha, \beta, \gamma, \delta)$ or $\left(\mu_{1}, \mu_{2}, \mu_{3}, \mu_{4}\right)$ depends on $\lambda$ and vanishes at $\lambda=0$.

PROOF: 43a is obtained by eliminating nonresonant terms and rescaling space and time. (43b) comes from the linear transformation $\eta \mapsto \eta-a \xi$ followed by a scaling. (43d) is obtained with the transformation $\xi \mapsto \xi+\frac{1}{3} \gamma$.

These normal forms describe equivalent codimension-four unfoldings of the singular vector field $\left(\dot{\xi}=\eta, \dot{\eta}=-\xi^{2} \eta-\xi^{3}\right)$. Indeed, the bifurcation is produced by setting four parameters to zero: the initial bifurcation parameter $\lambda$, the imaginary eigenvalue shift $\nu$, and the coefficients $v_{1}$ and $v_{2}$ of the feedback control.

Equation (43b) has been studied by Takens in the particular case $\gamma=\delta=0$ (which occurs when there is a symmetry $z \mapsto-z)$, who obtained the bifurcation diagram shown in Fig. 2 [Ta, GH]. A codimension-three unfolding corresponding to $\mu_{4}=0$ has been studied in $\mathrm{VT}$. The full codimension-four unfolding (43c) is discussed in KKR (see also references therein).

\section{Slowly Drifting Parameter}

Let us now consider the slowly time-dependent equation (8). We write $f(x, \lambda)+b u(x, \lambda)=$ $F(x, \lambda)$, where $u(x, \lambda)$ is the feedback control constructed in the previous section. The equation thus reads

$$
\varepsilon \frac{\mathrm{d} x}{\mathrm{~d} \tau}=F(x, \tau)
$$

By construction, $F(x, \tau)$ vanishes on the original equilibrium branch $x^{\star}(\tau)$. Let us write $F\left(x^{\star}(\tau)+x_{1}, \tau\right)=\hat{A}(\tau) x_{1}+\hat{g}\left(x_{1}, \tau\right)$, with $\hat{g}=\mathcal{O}\left(\left\|x_{1}\right\|^{2}\right)$. Hypothesis (H2) and the properties of $u$ imply that there exists an interval $\left[-T_{1}, 0\right)$ in which the matrix $\hat{A}(\tau)$ has only eigenvalues with a negative real part. Moreover, these real parts are bounded away from 0 on any interval $\left[\tau_{0}, \tau_{1}\right] \subset\left[-T_{1}, 0\right)$. It is well known (see for instance $[\mathrm{PR}]$ ) that this implies that any trajectory starting at $\tau_{0}$ in a sufficiently small neighbourhood of the origin reaches an $\mathcal{O}(\varepsilon)$-neighbourhood after a time of order $\varepsilon|\ln \varepsilon|$, where it remains until $\tau=\tau_{1}$ (this result is proved using Lyapunov functions). Hence it suffices to study (44) for $\tau \geqslant \tau_{1}$, with an initial condition $x\left(\tau_{1}\right)=\mathcal{O}(\varepsilon)$.

We first simplify equation (44) by applying similar transformations as for the timeindependent system, which will produce some additional terms of order $\varepsilon$. We will obtain an effective two-dimensional equation, which we will study by various methods in three different time intervals. 


\subsection{Reduction of the Equation}

The time-dependent translation $x=x^{\star}(\tau)+x_{1}$ yields the equation

$$
\varepsilon \frac{\mathrm{d} x_{1}}{\mathrm{~d} \tau}=\hat{A}(\tau) x_{1}+\hat{g}\left(x_{1}, \tau\right)-\varepsilon \frac{\mathrm{d} x^{\star}(\tau)}{\mathrm{d} \tau} .
$$

For small $\tau$, there exists a nonsingular matrix $S(\tau)$ such that $S^{-1} \hat{A} S$ is block-diagonal, with blocks $A_{-}$and $B$ as in (25) and (42). The transformation $x_{1}=S(\tau) x_{2}$ yields the equation

$$
\varepsilon \frac{\mathrm{d} x_{2}}{\mathrm{~d} \tau}=\left(\begin{array}{cc}
A_{-}(\tau) & 0 \\
0 & B(\tau)
\end{array}\right) x_{2}+S^{-1} \hat{g}\left(S x_{2}, \tau\right)-\varepsilon S^{-1}\left[\frac{\mathrm{d} S(\tau)}{\mathrm{d} \tau} x_{2}+\frac{\mathrm{d} x^{\star}(\tau)}{\mathrm{d} \tau}\right] .
$$

Next we apply a center manifold reduction. We write $x_{2}=\left(y_{2}, z_{2}\right) \in \mathbb{R}^{m} \times \mathbb{R}^{2}$.

Proposition 5.1. For sufficiently small $\varepsilon$, equation (46) admits a local invariant manifold (local in $z_{2}$ and $\tau$ ), with a parametric equation of the form $x_{2}=\left(h\left(z_{2}, \tau, \varepsilon\right), z_{2}\right)$, where $\left\|h\left(z_{2}, \tau, \varepsilon\right)\right\| \leqslant M\left(\left\|z_{2}\right\|^{2}+\tau^{2}+\varepsilon\right)$. Any solution starting at a distance of order $\varepsilon$ from this manifold is such that $y_{2}(\tau)=h\left(z_{2}(\tau), \tau, \varepsilon\right)+\mathcal{O}(\varepsilon)$ in some neighbourhood of $\left(x_{2}, \tau\right)=(0,0)$, where $z_{2}(\tau)$ satisfies the equation

$$
\varepsilon \frac{\mathrm{d} z_{2}}{\mathrm{~d} \tau}=B(\tau) z_{2}+G\left(z_{2}, \tau\right)+\varepsilon P\left(z_{2}, \tau, \varepsilon\right),
$$

with $B(\tau)$ and $G\left(z_{2}, \tau\right)$ the same functions as in (42). Moreover, the second component of $P(0,0,0)$ is different from 0 .

Proof: Equation (46) can also be written as

$$
\begin{aligned}
\mathrm{d}_{t} y_{2} & =A_{-}(\tau) y_{2}+\varepsilon w_{-}(\tau)+\mathcal{O}\left(\left\|y_{2}\right\|^{2}+\left\|z_{2}\right\|^{2}+\tau^{2}+\varepsilon^{2}\right) \\
\mathrm{d}_{t} z_{2} & =B(\tau) z_{2}+\varepsilon w_{0}(\tau)+\mathcal{O}\left(\left\|y_{2}\right\|^{2}+\left\|z_{2}\right\|^{2}+\tau^{2}+\varepsilon^{2}\right) \\
\mathrm{d}_{t} \tau & =\varepsilon \\
\mathrm{d}_{t} \varepsilon & =0 .
\end{aligned}
$$

The transformation $y_{2}=y_{3}-\varepsilon A_{-}^{-1}(0) w_{-}(0)$ yields a system whose linearization at the point $\left(y_{3}, z_{2}, \tau, \varepsilon\right)=(0,0,0,0)$ is the matrix

$$
\left(\begin{array}{cccc}
A_{-}(0) & 0 & 0 & 0 \\
0 & B(0) & 0 & w_{0}(0) \\
0 & 0 & 0 & 1 \\
0 & 0 & 0 & 0
\end{array}\right) .
$$

The center manifold theorem implies the existence of a local invariant manifold $y_{3}=$ $\tilde{h}\left(z_{2}, \tau, \varepsilon\right)=\mathcal{O}\left(\left\|z_{2}\right\|^{2}+\tau^{2}+\varepsilon^{2}\right)$. Moreover, it is shown in Lemma 1, p. 20 of [Ca], that in some neighbourhood of the origin, any solution satisfies a bound of the form

$$
\left\|y_{3}(\tau)-h\left(z_{2}(\tau), \tau, \varepsilon\right)\right\| \leqslant M \mathrm{e}^{-\kappa\left(\tau-\tau_{0}\right) / \varepsilon}\left\|y_{3}\left(\tau_{0}\right)-h\left(z_{2}\left(\tau_{0}\right), \tau, \varepsilon\right)\right\|,
$$

for some positive $M, \kappa$. Since $\left\|y_{3}\left(\tau_{0}\right)-h\left(z_{2}\left(\tau_{0}\right), \tau, \varepsilon\right)\right\|=\mathcal{O}(\varepsilon)$, we have

$$
\varepsilon \frac{\mathrm{d} z_{2}}{\mathrm{~d} \tau}=B(\tau) z_{2}+g_{0}\left(h\left(z_{2}, \tau, \varepsilon\right)+\mathcal{O}(\varepsilon), z_{2}, \tau, \varepsilon\right) .
$$

When $\varepsilon=0$, this equation coincides with the equation on the instantaneous center manifold. The assertion on $P(0,0,0)$ follows from Hypothesis $(\mathrm{H} 5)$. 
As a final reduction step, we may apply a nonlinear transformation putting the 3 -jet of $G\left(z_{2}, \tau\right)$ into one of the canonical forms (43). This will produce terms of order $\varepsilon\left\|z_{2}\right\|^{2}$, that we may also absorb into the remainder $P\left(z_{2}, \tau, \varepsilon\right)$. A redefinition of the variable $\eta$ yields the canonical form (11). In the sequel, we will use the equivalent form

$$
\begin{aligned}
\varepsilon \dot{\xi} & =a(\tau) \xi+\eta \\
\varepsilon \dot{\eta} & =\mu(\tau) \xi+a(\tau) \eta+\hat{\gamma}(\tau) \xi^{2}+\hat{\delta}(\tau) \xi \eta-\xi^{3}-\xi^{2} \eta+\mathcal{O}\left(\|z\|^{4}\right)+\varepsilon R(\xi, \eta, \tau, \varepsilon),
\end{aligned}
$$

where we now use the dots to indicate the derivative with respect to $\tau$. We can rescale time in such a way that

$$
a(\tau)=c \tau+\mathcal{O}\left(\tau^{2}\right), \quad \mu(\tau)=\tau+\mathcal{O}\left(\tau^{2}\right), \quad \hat{\gamma}(\tau)=\mathcal{O}(\tau), \quad \hat{\delta}(\tau)=\mathcal{O}(\tau),
$$

where $c=\mathrm{d} a / \mathrm{d} \mu(0)=\dot{a}(0) /(C-\dot{\omega}(0))$, see (41).

We will study equation (52) by different methods in three different regions. They are characterized by a constant $d$ which will be chosen sufficiently small, but is independent of $\varepsilon$. These regions are:

1. Before the bifurcation: $\tau_{1}(\varepsilon) \leqslant \tau \leqslant-(\varepsilon / d)^{2 / 3}$, where $\tau_{1}(\varepsilon)=\tau_{0}+\mathcal{O}(\varepsilon \ln \varepsilon)$ is chosen in such a way that $z\left(\tau_{1}\right)=\mathcal{O}(\varepsilon)$. In this region (52) can be transformed into a onedimensional complex equation, that we study by suitable normal form transformations. The main difficulty is to use the averaging effect of fast oscillations around the focus.

2. During the bifurcation: $-(\varepsilon / d)^{2 / 3} \leqslant \tau \leqslant(\varepsilon / d)^{2 / 3}$. In this inner region, (52) can be reduced to a time-dependent Hamiltonian system by an appropriate scaling.

3. After the bifurcation: $(\varepsilon / d)^{2 / 3} \leqslant \tau \leqslant T$, where $T$ will be chosen sufficiently small. By choosing $c$ in an appropriate way, we ensure the existence of an attracting equilibrium branch.

\subsection{Before the Bifurcation}

In the first region, we use a coordinate transformation which diagonalizes the linear part approximately. It is given by

$$
\zeta=\frac{1}{\sqrt{2}}\left[\mathrm{e}^{\mathrm{i} \pi / 4}|\mu|^{1 / 4} \xi+\mathrm{e}^{-\mathrm{i} \pi / 4}|\mu|^{-1 / 4} \eta\right]
$$

and its inverse reads

$$
\begin{aligned}
& \xi=\frac{1}{\sqrt{2}}\left[\mathrm{e}^{-\mathrm{i} \pi / 4} \zeta+\mathrm{e}^{\mathrm{i} \pi / 4} \bar{\zeta}\right]|\mu|^{-1 / 4} \\
& \eta=\frac{1}{\sqrt{2}}\left[\mathrm{e}^{\mathrm{i} \pi / 4} \zeta+\mathrm{e}^{-\mathrm{i} \pi / 4} \bar{\zeta}\right]|\mu|^{1 / 4}
\end{aligned}
$$

In these variables, equation (52) becomes

$$
\varepsilon \dot{\zeta}=\rho(\tau) \zeta+\varepsilon \varphi(\tau) \bar{\zeta}+\varepsilon w(\tau)+G_{0}(\zeta, \bar{\zeta}, \tau, \varepsilon)
$$

where

$$
\begin{aligned}
& \rho(\tau)=a(\tau)+\mathrm{i} \sqrt{-\mu(\tau)}+\varepsilon \psi(\tau), \quad \psi(\tau)=\mathcal{O}\left(|\tau|^{-1 / 2}\right) \\
& \varphi(\tau)=\mathrm{i} \dot{\mu}(\tau) / 4 \mu(\tau)=\mathcal{O}\left(|\tau|^{-1}\right), \\
& w(\tau)=\frac{1}{\sqrt{2}} \mathrm{e}^{-\mathrm{i} \pi / 4}|\mu|^{-1 / 4} R(0,0, \tau, 0)=\mathcal{O}\left(|\tau|^{-1 / 4}\right) \\
& G_{0}(\zeta, \bar{\zeta}, \tau, \varepsilon)=\mathcal{O}\left(|\tau|^{1 / 4}|\zeta|^{2}\right)+\mathcal{O}\left(|\tau|^{-1}|\zeta|^{3}\right)+\mathcal{O}\left(|\tau|^{-5 / 4}|\zeta|^{4}\right) .
\end{aligned}
$$

The main result of this subsection is the following estimate. 
Proposition 5.2. If $d$ and $\varepsilon$ are sufficiently small, there exists a constant $M_{1}>0$ such that equation (56) admits a particular solution $\zeta_{0}(\tau)$ satisfying

$$
\left|\zeta_{0}(\tau)+\varepsilon \frac{w(\tau)}{a(\tau)+\mathrm{i} \sqrt{-\mu(\tau)}}\right| \leqslant M_{1} \frac{\varepsilon^{2}}{|\tau|^{9 / 4}} \quad \text { for } \tau_{1} \leqslant \tau \leqslant-(\varepsilon / d)^{2 / 3} .
$$

Moreover, any solution of (56) with initial condition $\zeta\left(\tau_{1}\right)=\mathcal{O}(\varepsilon)$ satisfies the bound

$$
\left|\zeta(\tau)-\zeta_{0}(\tau)\right| \leqslant M_{2} \varepsilon \mathrm{e}^{-\kappa\left(\tau^{2}-\tau_{1}^{2}\right) / 2 \varepsilon}
$$

on the same time interval, for some positive constants $M_{2}, \kappa$.

Corollary 5.3. Any solution of (52) with initial condition of order $\varepsilon$ satisfies

$$
\xi(\tau)=\mathcal{O}\left(\varepsilon|\tau|^{-1}\right), \quad \eta(\tau)=\mathcal{O}\left(\varepsilon|\tau|^{-1 / 2}\right)
$$

on the interval $\tau_{1} \leqslant \tau \leqslant-(\varepsilon / d)^{2 / 3}$. In particular, at $\tau=-(\varepsilon / d)^{2 / 3}$ we have

$$
\begin{aligned}
\xi & =d^{2 / 3} \varepsilon^{1 / 3}\left[R(0,0,0,0)+\mathcal{O}(d)+\mathcal{O}\left((\varepsilon / d)^{1 / 3}\right)\right], \\
\eta & =d^{1 / 3} \varepsilon^{2 / 3}\left[\mathcal{O}(d)+\mathcal{O}\left((\varepsilon / d)^{1 / 3}\right)\right] .
\end{aligned}
$$

Remark 5.4. One can in fact show the existence of a particular solution admitting an asymptotic series of the form

$$
\zeta_{0}(\tau)=\frac{\varepsilon}{|\tau|^{3 / 4}}\left[c_{0}(\tau)+c_{1}(\tau) \frac{\varepsilon}{|\tau|^{3 / 2}}+c_{2}(\tau) \frac{\varepsilon^{2}}{|\tau|^{3}}+\cdots\right]
$$

For $\tau=(\varepsilon / d)^{2 / 3}$, we get an asymptotic series in $d$. Proposition 5.2 is only a first step, but suffices for our purposes.

The proof of Proposition 5.2 is based on the following two lemmas. The first one is a rather trivial, but very useful bound on an integral we will encounter several times, while the second one gives an "a priori" estimate on $\zeta_{0}(\tau)$.

Lemma 5.5. Assume that we are given

- constants $\tau_{1} \leqslant \tau<0, a_{0}, w_{0}>0$ and $p, q$ such that $p+1-q>0$.

- $\Psi(\tau):\left[\tau_{1}, 0\right) \rightarrow \mathbb{C}$ differentiable such that $\operatorname{Re} \dot{\Psi}(\tau) \leqslant 0$ and $|\dot{\Psi}(\tau)| \geqslant|\tau|^{p} / a_{0}$.

- $w(\tau):\left[\tau_{1}, 0\right) \rightarrow \mathbb{C}$ differentiable with $|w(\tau)| \leqslant w_{0}|\tau|^{q-1}$ and $|\dot{w}(\tau)| \leqslant w_{0}|\tau|^{q-2}$.

Then

$$
\left|\mathrm{e}^{\Psi(\tau) / \varepsilon} \int_{\tau_{1}}^{\tau} \mathrm{e}^{-\Psi(s) / \varepsilon} w(s) \mathrm{d} s\right| \leqslant \frac{\varepsilon}{|\tau|^{p+1-q}} K, \quad K=a_{0} w_{0}\left[2+\frac{1}{p+1-q}\right] .
$$

PROOF: Use integration by parts once.

Lemma 5.6. Let $c_{0}>0$ be given. If $\varepsilon$ and $d$ are small enough, there exists a constant $M_{1}\left(c_{0}\right)$ such that any solution of (56) with initial condition $\left|\zeta\left(\tau_{1}\right)\right| \leqslant c_{0} \varepsilon$ satisfies

$$
|\zeta(\tau)| \leqslant M_{1} \frac{\varepsilon}{|\tau|^{3 / 4}} \quad \text { for } \tau_{1} \leqslant \tau \leqslant-(\varepsilon / d)^{2 / 3} .
$$


Proof: Notice that for small enough $d$, we have $|\rho| \geqslant K_{0}|\tau|^{1 / 2}$.

- Step 1: Simplification of the linear part.

Consider the initial value problem

$$
\varepsilon \dot{s}=[\rho(\tau)-\bar{\rho}(\tau)] s+\varepsilon \varphi(\tau)-\varepsilon \bar{\varphi}(\tau) s^{2}, \quad s\left(\tau_{1}\right)=0 .
$$

Since $\mathrm{d}_{\tau}|s|^{2}=(\varphi \bar{s}-\bar{\varphi} s)\left(1-|s|^{2}\right)$, its solution satisfies $|s(\tau)| \leqslant 1$. (In fact, one can prove that $\left.s=\mathcal{O}\left(\varepsilon|\tau|^{-3 / 2}\right)\right)$. The transformation $\zeta=\zeta_{1}+s(\tau) \bar{\zeta}_{1}$ yields

$$
\varepsilon \dot{\zeta}_{1}=\rho_{1}(\tau) \zeta_{1}+\varepsilon w_{1}(\tau)+G_{1}\left(\zeta_{1}, \bar{\zeta}_{1}, \tau, \varepsilon\right),
$$

where $\rho_{1}=\rho+\varepsilon \varphi \bar{s}$ satisfies $\left|\rho_{1}\right| \geqslant K_{1}|\tau|^{1 / 2}$, and $G_{1}$ and $w_{1}$ satisfy similar bounds as $G_{0}$ and $w$.

- Step 2: Simplification of the cubic part.

Let us consider the effect of a change of variables

$$
\zeta_{1}=\zeta_{2}+h\left(\zeta_{2}, \bar{\zeta}_{2}, \tau\right), \quad h\left(\zeta_{2}, \bar{\zeta}_{2}, \tau\right)=\sum_{n+m=3} h_{n m}(\tau) \zeta_{2}^{n} \bar{\zeta}_{2}^{m}
$$

It transforms (66) into

$$
\begin{aligned}
\varepsilon \dot{\zeta}_{2}=\rho_{1} \zeta_{2}+\varepsilon w_{1}- & \varepsilon \partial_{\zeta} h w_{1}-\varepsilon \partial_{\bar{\zeta}} h \bar{w}_{1}+G_{1}^{(2)}+ \\
& +\left[G_{1}^{(3)}+\rho_{1} h-\partial_{\zeta} h \rho_{1} \zeta_{2}-\partial_{\bar{\zeta}} h \bar{\rho}_{1} \bar{\zeta}_{2}-\varepsilon \partial_{\tau} h\right]+\mathcal{O}\left(\left\|z_{2}\right\|^{4}\right),
\end{aligned}
$$

where $G_{1}^{(k)}$ denotes terms of order $k$. In particular,

$$
G_{1}^{(3)}\left(\zeta_{2}, \bar{\zeta}_{2}, \tau\right)=\sum_{n+m=3} g_{n m}(\tau) \zeta_{2}^{n} \bar{\zeta}_{2}^{m}
$$

with $g_{n m}(\tau)=\mathcal{O}\left(|\tau|^{-1}\right)$. We see that the term in brackets of (68) can be eliminated if

$$
\varepsilon \dot{h}_{n m}=\left[(1-n) \rho_{1}-m \bar{\rho}_{1}\right] h_{n m}+g_{n m} .
$$

This is a linear equation which can easily be solved. If $(n, m) \neq(2,1)$, one can choose the initial condition in such a way that $\left|h_{n m}(\tau)\right| \leqslant K_{2}|\tau|^{-3 / 2}$. When $(n, m)=(2,1)$, however, we cannot obtain such a good bound because the imaginary part of the term in brackets vanishes. Thus we do not attempt to eliminate this term. We obtain the equation

$$
\varepsilon \dot{\zeta}_{2}=\left[\rho_{1}(\tau)-g_{21}(\tau)\left|\zeta_{2}\right|^{2}\right] \zeta_{2}+\varepsilon w_{1}(\tau)+G_{2}\left(\zeta_{2}, \bar{\zeta}_{2}, \tau, \varepsilon\right),
$$

where

$$
\left|G_{2}\left(\zeta_{2}, \bar{\zeta}_{2}, \tau, \varepsilon\right)\right| \leqslant M_{2}\left[|\tau|^{1 / 4}\left|\zeta_{2}\right|^{2}+\varepsilon|\tau|^{-7 / 4}\left|\zeta_{2}\right|^{2}+|\tau|^{-5 / 4}\left|\zeta_{2}\right|^{4}\right] .
$$

Let us write $\rho_{2}\left(\tau, \zeta_{2}\right)=\rho_{1}(\tau)-g_{21}(\tau)\left|\zeta_{2}\right|^{2}$. 
- Step 3: Proof of the bound (64).

The solution of (71) has to satisfy

$$
\begin{aligned}
& \zeta_{2}(\tau)=\mathrm{e}^{\left[\Psi_{2}(\tau)-\Psi_{2}\left(\tau_{1}\right)\right] / \varepsilon} \zeta_{2}\left(\tau_{0}\right)+ \\
& \quad \mathrm{e}^{\Psi_{2}(\tau) / \varepsilon} \int_{\tau_{1}}^{\tau} \mathrm{e}^{-\Psi_{2}(\tau) / \varepsilon}\left[w_{1}(\tau)+\frac{1}{\varepsilon} G_{2}\left(\zeta_{2}, \bar{\zeta}_{2}, \tau, \varepsilon\right)\right] \mathrm{d} s,
\end{aligned}
$$

where $\Psi_{2}(\tau)=\int_{0}^{\tau} \rho_{2}\left(s, \zeta_{2}(s)\right) \mathrm{d} s$. We define the time

$$
\tau^{\star}=\sup _{\left[\tau_{1},-(\varepsilon / d)^{2 / 3}\right]}\left\{\tau|| \zeta_{2}(s) \mid \leqslant \sqrt{\varepsilon} \text { for } \tau_{1} \leqslant s \leqslant \tau\right\} .
$$

By continuity, $\tau^{\star}>\tau_{1}$. Using the bounds on $G_{2}$ we can show that for $\tau_{1} \leqslant \tau \leqslant \tau^{\star}$,

$$
\begin{aligned}
\left|\rho_{2}\left(\tau, \zeta_{2}\right)\right| & \geqslant K_{2}|\tau|^{1 / 2} \\
\left|G_{2}\right| & \leqslant M_{3}\left[\varepsilon|\tau|^{1 / 4}+\varepsilon^{2}|\tau|^{-7 / 4}\right], \\
\left|\mathrm{d}_{\tau} G_{2}\right| & \leqslant M_{3}\left[\varepsilon|\tau|^{-5 / 4}+\varepsilon^{2}|\tau|^{-11 / 4}\right],
\end{aligned}
$$

where $\mathrm{d}_{\tau} G_{2}=\partial_{\tau} G_{2}+\partial_{\zeta_{2}} G_{2} \dot{\zeta}_{2}+\partial_{\bar{\zeta}_{2}} G_{2} \dot{\bar{\zeta}}_{2}$ is estimated using (71). Using Lemma 5.5 and the bounds on $w_{1}$, we obtain that

$$
\begin{aligned}
\left|\zeta_{2}(\tau)\right| & \leqslant M_{4}\left[\varepsilon|\tau|^{-3 / 4}+\varepsilon^{2}|\tau|^{-9 / 4}\right] \\
\Rightarrow \quad\left|\zeta_{2}\left(\tau^{\star}\right)\right| & \leqslant M_{4} \varepsilon^{1 / 2}\left[d^{1 / 2}+d^{3 / 2}\right] .
\end{aligned}
$$

Taking $d$ sufficiently small, we have $\left|\zeta_{2}\left(\tau^{\star}\right)\right|<\varepsilon^{1 / 2}$. If we assume that $\tau^{\star}<-(\varepsilon / d)^{2 / 3}$, we contradict the definition of $\tau^{\star}$, which shows that $\tau^{\star}=-(\varepsilon / d)^{2 / 3}$.

Going back to the initial variables and using the bounds on $s$ and $h$, we obtain the conclusion of the lemma.

Proof of Proposition 5.2. After subtracting $\varepsilon w(\tau) / \rho(\tau)$ from (56) and eliminating the term linear in $\bar{\zeta}$ in the same way as in Lemma 5.6, we obtain

$$
\varepsilon \dot{\zeta}_{1}=\rho_{1}(\tau) \zeta_{1}+\varepsilon^{2} w_{1}(\tau)+G_{1}\left(\zeta_{1}, \bar{\zeta}_{1}, \tau, \varepsilon\right)
$$

where

$$
\begin{aligned}
\left|\rho_{1}(\tau)\right| & \geqslant K_{1}|\tau|^{1 / 2} \\
w_{1}(\tau) & =\mathcal{O}\left(|\tau|^{-7 / 4}\right), \\
G_{1}\left(\zeta_{1}, \bar{\zeta}_{1}, \tau, \varepsilon\right) & =\mathcal{O}\left(|\tau|^{1 / 4}\left|\zeta_{1}\right|^{2}\right)+\mathcal{O}\left(\varepsilon|\tau|^{-7 / 4}\left|\zeta_{1}\right|^{2}\right)+\mathcal{O}\left(|\tau|^{-1}\left|\zeta_{1}\right|^{3}\right) .
\end{aligned}
$$

Let $\Psi_{1}(\tau)$ be a primitive of $\rho_{1}(\tau)$. The solution of $(77)$ with initial condition $\zeta_{1}\left(\tau_{1}\right)=0$ should satisfy

$$
\zeta_{1}(\tau)=\mathrm{e}^{\Psi_{1}(\tau) / \varepsilon} \int_{\tau_{1}}^{\tau} \mathrm{e}^{-\Psi_{1}(s) / \varepsilon}\left[\varepsilon w_{1}(s)+\frac{1}{\varepsilon} G_{1}\left(\zeta_{1}, \bar{\zeta}_{1}, s, \varepsilon\right)\right] \mathrm{d} s .
$$

Using the a priori estimate of Lemma 5.6 and the bounds (78), we can apply Lemma 5.5 to estimate the integral and obtain (58). To obtain (59), it is sufficient to subtract the particular solution $\zeta_{0}(\tau)$ from the general solution, and to use the modulus as a Lyapunov function. 


\subsection{During the Bifurcation}

In this subsection, we study (52) on the time interval $\left[-(\varepsilon / d)^{2 / 3},(\varepsilon / d)^{2 / 3}\right]$. We recall that $d$ is a constant which will be chosen small, but is independent of $\varepsilon$, while $\varepsilon$ is small with respect to $d$. In other words, we consider the system on a time scale $d^{-1}$, which is intermediate between 1 and $\varepsilon^{-1}$. In fact it turns out to be useful to take $\varepsilon \leqslant d^{4}$.

From Corollary 5.3, we know that at the time $\tau=-(\varepsilon / d)^{2 / 3}, \xi$ is of order $\varepsilon^{1 / 3}$ and $\eta$ is of order $\varepsilon^{2 / 3}$. The basic idea to analyse the motion during the bifurcation is to introduce the scaling of variables

$$
\xi=(\varepsilon / d)^{1 / 3} x, \quad \eta=(\varepsilon / d)^{2 / 3} y, \quad \tau=(\varepsilon / d)^{2 / 3} t .
$$

This scaling transforms the system (52) into

$$
\begin{aligned}
& d \dot{x}=y+(\varepsilon / d)^{1 / 3} Q_{1}(x, y, t, \varepsilon), \\
& d \dot{y}=t x-x^{3}+d R_{0}+(\varepsilon / d)^{1 / 3} Q_{2}(x, y, t, \varepsilon),
\end{aligned}
$$

where $R_{0}=R(0,0,0,0)$ and the functions $Q_{1}$ and $Q_{2}$ are uniformly bounded by constants independent of $\varepsilon$ and $d$. This system should be studied on the time interval $t \in[-1,1]$, and with an initial condition

$$
\begin{aligned}
& x(-1)=d\left[R_{0}+\mathcal{O}(d)+\mathcal{O}\left((\varepsilon / d)^{1 / 3}\right)\right]=d R_{0}+\mathcal{O}\left(d^{2}\right), \\
& y(-1)=d\left[\mathcal{O}(d)+\mathcal{O}\left((\varepsilon / d)^{1 / 3}\right)\right]=\mathcal{O}\left(d^{2}\right) .
\end{aligned}
$$

Equation (81) is a small perturbation of the Hamiltonian system

$$
H(x, y, t)=\frac{1}{d}\left[\frac{1}{2} y^{2}+\frac{1}{4} x^{4}-\frac{1}{2} t x^{2}-d R_{0} x\right] .
$$

Lemma 5.7. For $-1 \leqslant t \leqslant 1$, the solution of equation (81) and of the Hamiltonian system (83) with the same initial condition differ by a term of order $(\varepsilon / d)^{1 / 3}$.

Proof: One can use a standard averaging result, see for instance [GH], Theorem 4.1.1. page 168 .

The Hamiltonian (83) describes the motion of a particle in a potential $V(x, t)=\frac{1}{4} x^{4}-$ $\frac{1}{2} t x^{2}-d R_{0} x$ which changes from a single well to a double well as $t$ grows from -1 to 1 (Fig. 3). Note that (82) implies that the energy is negative at $t=-1$ if $d$ is sufficiently small. Since

$$
\frac{\mathrm{d} H}{\mathrm{~d} t}(x, y, t)=\frac{\partial H}{\partial t}(x, y, t)=-\frac{1}{2 d} x(t)^{2},
$$

the energy must decrease. Since the barrier between the potential wells always has positive energy, the particle has to remain in the same well, which is the right one if $R_{0}>0$ and the left one if $R_{0}<0$.

We will however need a more precise estimate, showing that the orbit remains close to the bottom of the well. This is the main result of this section.

Proposition 5.8. Assume that $R_{0} \neq 0$. For sufficiently small $d$, the solution of the Hamiltonian system (83) with the initial condition (82) is such that

$$
x(1)=\operatorname{sign} R_{0}+\mathcal{O}(d), \quad y(1)=\mathcal{O}(d) .
$$



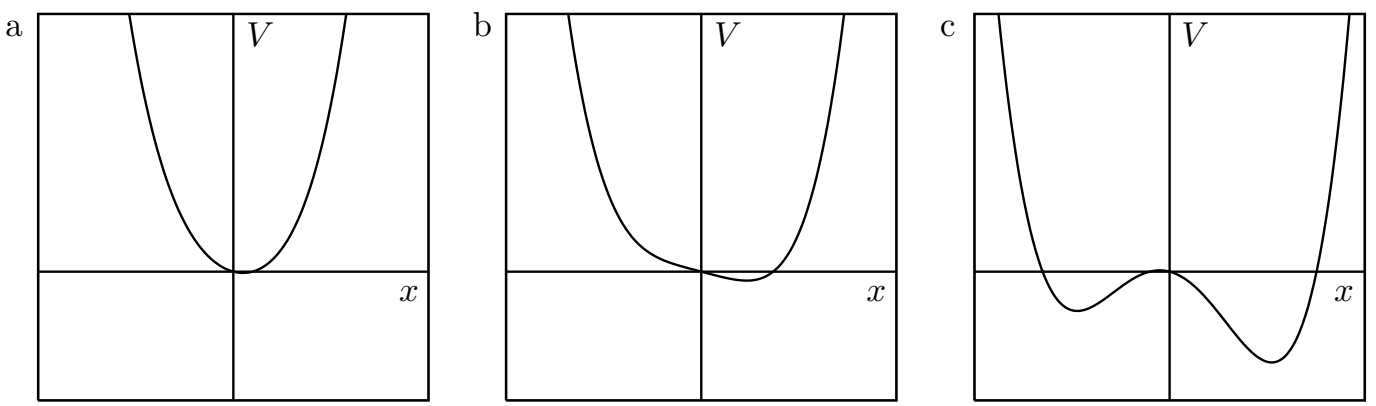

FiguRE 3. The dynamics near the bifurcation point can be reduced to the motion of a particle in a time-dependent potential which transforms from a single to a double well. The potential $V(x, t)=\frac{1}{4} x^{4}-\frac{1}{2} t x^{2}-d R_{0} x$ is shown for $d R_{0}=0.1$ and $t=-1,0$ and 1. Since the energy is decreasing, and the barrier between the wells always has a positive energy, the particle remains in the same well.

Together with Lemma 5.7, this implies

Corollary 5.9. During the time interval $\left[-(\varepsilon / d)^{2 / 3},(\varepsilon / d)^{2 / 3}\right]$, the solution of (52) with initial condition given by Corollary 5.3 satisfies $\xi=\mathcal{O}\left(\varepsilon^{1 / 3}\right)$ and $\eta=\mathcal{O}\left(\varepsilon^{2 / 3}\right)$. At the time $\tau=(\varepsilon / d)^{2 / 3}$ we have

$$
\begin{aligned}
& \xi=(\varepsilon / d)^{1 / 3} \operatorname{sign} R_{0}+\mathcal{O}\left(\varepsilon^{1 / 3} d^{2 / 3}\right)+\mathcal{O}\left((\varepsilon / d)^{2 / 3}\right), \\
& \eta=\mathcal{O}\left(\varepsilon^{2 / 3} d^{1 / 3}\right)+\mathcal{O}(\varepsilon / d) .
\end{aligned}
$$

Proof of Proposition 5.8. To simplify the notation, we consider the case $R_{0}=1$.

- Step 1: Transformation of the equation.

Let $x_{\star}(t)$ be the positive solution of $t x_{\star}-x_{\star}^{3}+d=0$. Then we have

$$
\begin{aligned}
& \dot{x}_{\star}(t)=\frac{x_{\star}(t)}{a(t)}, \quad \ddot{x}_{\star}(t)=\frac{2 t x_{\star}(t)}{a(t)^{2}}, \\
& a(t)=3 x_{\star}^{2}-t \geqslant \max \left\{-t, 2 d^{2 / 3}, 2 t\right\} .
\end{aligned}
$$

We decrease the order of the drift term $d$ by the transformation

$$
\begin{aligned}
& x=x_{1}+x_{\star}(t)-d^{2} \frac{\ddot{x}_{\star}}{a} \\
& y=y_{1}+d \dot{x}_{\star}(t)-d^{3} \frac{\mathrm{d}}{\mathrm{d} t}\left(\frac{\ddot{x}_{\star}}{a}\right),
\end{aligned}
$$

which implies that $x_{1}(-1), y_{1}(-1)=\mathcal{O}\left(d^{2}\right)$ and yields the system

$$
\begin{aligned}
& d \dot{x}_{1}=y_{1} \\
& d \dot{y}_{1}=-a_{1}(t) x_{1}-b_{1}(t) x_{1}^{2}-x_{1}^{3}+d^{4} c_{1}(t),
\end{aligned}
$$

where

$$
\begin{aligned}
a_{1}(t) & =a(t)+\mathcal{O}\left(d^{4 / 3}\right), \\
b_{1}(t) & =3 x_{\star}(t)+\mathcal{O}(d), \\
c_{1}(t) & =\frac{\mathrm{d}^{2}}{\mathrm{~d} t^{2}}\left(\frac{\ddot{x}_{\star}}{a}\right)+\mathcal{O}\left(d^{-5 / 3}\right) .
\end{aligned}
$$


There is a time $t_{\star}=\mathcal{O}\left(d^{2 / 3}\right)$ such that $\dot{a}_{1}(t)<0$ for $t<t_{\star}$ and $\dot{a}_{1}(t)>0$ for $t>t_{\star}$. Using (87) and some algebra, we obtain the existence of a constant $M>0$ such that

$$
\frac{\left|b_{1}(t)\right|}{M} \leqslant\left\{\begin{array}{l}
d|t|^{-1} \\
d^{2 / 3} \\
t^{1 / 2},
\end{array} \quad \frac{\left|c_{1}(t)\right|}{M} \leqslant\left\{\begin{array} { l } 
{ d | t | ^ { - 4 } } \\
{ d ^ { - 5 / 3 } } \\
{ t ^ { - 5 / 2 } , }
\end{array} \quad \text { for } \left\{\begin{array}{l}
t \leqslant-d^{2 / 3} \\
|t| \leqslant d^{2 / 3} \\
t \geqslant d^{2 / 3}
\end{array}\right.\right.\right.
$$

- Step 2: $t \leqslant t_{\star}$.

Consider the Lyapunov function

$$
V=\left[\frac{1}{2} y_{1}^{2}+\frac{1}{2} a_{1}(t) x_{1}^{2}+\frac{1}{4} x_{1}^{4}\right]^{1 / 2} .
$$

We have $V(-1) \leqslant M_{0} d^{2}$ and

$$
d \dot{V}=\frac{1}{2 V}\left[\frac{1}{2} d \dot{a}_{1} x_{1}^{2}-b_{1} x_{1}^{2} y_{1}+d^{4} c_{1} y_{1}\right] \leqslant 2^{-1 / 2} d^{4}\left|c_{1}\right|+2^{1 / 2} \frac{b_{1}}{a_{1}} V^{2} .
$$

From (92) we get the bound

$$
\int_{-1}^{t_{\star}} 2^{-1 / 2} d^{3}\left|c_{1}(s)\right| \mathrm{d} s \leqslant M_{1} d^{2} .
$$

Let $M_{2}=M_{0}+2 M_{1}$ and define the time

$$
\hat{t}=\sup _{-1 \leqslant t \leqslant t_{\star}}\left\{t \mid V(s)<M_{2} d^{2} \text { for }-1 \leqslant s<t\right\} .
$$

For $t \leqslant \hat{t}$ we get from (94) and a standard result on differential inequalities (see for instance $\mathrm{Hal}$ ) that

$$
V(t) \leqslant\left(M_{0}+M_{1}\right) d^{2}+M_{3} M_{2}^{2} d^{10 / 3}<M_{2} d^{2}
$$

for sufficiently small $d$, which proves that $\hat{t}=t_{\star}$ and thus $V\left(t_{\star}\right)=\mathcal{O}\left(d^{2}\right)$.

- Step 3: $t \geqslant t_{\star}$.

Using the fact that $\dot{a}_{1}>0$ we obtain

$$
d \dot{V} \leqslant \frac{1}{2} d \frac{\dot{a}_{1}}{a_{1}} V+2^{-1 / 2} d^{4}\left|c_{1}\right|+2^{1 / 2} \frac{b_{1}}{a_{1}} V^{2} .
$$

We choose $M_{4}$ such that $M_{2} d^{1 / 3}<M_{4}$ and define

$$
\tilde{t}=\sup _{t_{\star} \leqslant t \leqslant 1}\left\{t \mid V(s)<M_{4} d^{5 / 3} \text { for } t_{\star} \leqslant s<t\right\} .
$$

For $t \leqslant \tilde{t}$, we have

$$
\dot{V} \leqslant \frac{\dot{a}_{1}}{2 a_{1}}\left[1+M M_{4} d^{2 / 3}\right] V+2^{-1 / 2} d^{3}\left|c_{1}\right| .
$$

Using Gronwall's Lemma, we obtain that if $M M_{4} d^{2 / 3} \ln \left[a_{1}(1) / a_{1}\left(t_{\star}\right)\right] \leqslant \ln 2$,

$$
V(t) \leqslant 2\left[\frac{a_{1}(t)}{a_{1}\left(t_{\star}\right)}\right]^{1 / 2} V\left(t_{\star}\right)+\left[2 a_{1}(t)\right]^{1 / 2} \int_{t_{\star}}^{t} d^{3} \frac{\left|c_{1}(s)\right|}{a_{1}(s)^{1 / 2}} \mathrm{~d} s \leqslant M_{5} d^{2 / 3} .
$$

Taking $M_{4}>M_{5}$, we obtain $\tilde{t}=1$ and thus $V(1)=\mathcal{O}\left(d^{5 / 3}\right)$. Transforming back to the original variables, we obtain $x(1)=x_{\star}(1)+\mathcal{O}\left(d^{5 / 3}\right)$ and $y(1)=d \dot{x}_{\star}(1)+\mathcal{O}\left(d^{5 / 3}\right)$. 


\subsection{After the Bifurcation}

In this section, we analyse equation (52) for $\tau \geqslant(\varepsilon / d)^{2 / 3}$. It is in fact more convenient to use the form

$$
\begin{aligned}
& \varepsilon \dot{\xi}=\eta \\
& \varepsilon \dot{\eta}=\bar{\mu}(\tau) \xi+2 a(\tau) \eta+\gamma(\tau) \xi^{2}+\delta(\tau) \xi \eta-\xi^{3}-\xi^{2} \eta+\mathcal{O}\left(\|z\|^{4}\right)+\varepsilon R(\xi, \eta, \tau, \varepsilon),
\end{aligned}
$$

obtained by the transformation $\eta \mapsto \eta-a(\tau) \xi$, where $\bar{\mu}=\mu-a^{2}$. The right-hand side vanishes approximately on three curves $(\xi, \eta) \equiv(0,0)$ and $(\xi, \eta)=\left(\xi_{ \pm}^{\star}(\tau), 0\right)$, where

$$
\xi_{ \pm}^{\star}(\tau)= \pm \bar{\mu}^{1 / 2}+\mathcal{O}(\tau)= \pm \tau^{1 / 2}+\mathcal{O}(\tau)
$$

We assume here that $R(0,0,0,0)>0$ so that the initial condition is close to $\xi_{+}^{\star}$, the other case is obtained by symmetry. The translation $\xi=\xi_{+}^{\star}(\tau)+\xi_{1}, \eta=\eta_{1}$ yields

$$
\begin{aligned}
& \varepsilon \dot{\xi_{1}}=\eta_{1} \\
& \varepsilon \dot{\eta_{1}}=-\beta \xi_{1}+2 \alpha \eta_{1}+\tilde{\gamma} \xi_{1}^{2}+\tilde{\delta} \xi_{1} \eta_{1}-\xi_{1}^{3}-\xi_{1}^{2} \eta_{1}+\mathcal{O}\left(\left\|z_{1}\right\|^{4}\right)+\varepsilon R,
\end{aligned}
$$

where (see equation (53))

$$
\begin{aligned}
\alpha(\tau) & =a-\frac{1}{2} \bar{\mu}+\frac{1}{2}(\gamma-\delta) \xi_{+}^{\star}=\left(c-\frac{1}{2}\right) \tau+\mathcal{O}\left(\tau^{3 / 2}\right), \\
\beta(\tau) & =2 \xi_{+}^{\star}-\delta \xi_{+}^{\star}=2 \tau+\mathcal{O}\left(\tau^{3 / 2}\right), \\
\tilde{\gamma}(\tau) & =\gamma-2 \xi_{+}^{\star}=-2 \tau^{1 / 2}+\mathcal{O}(\tau), \\
\tilde{\delta}(\tau) & =\delta-3 \xi_{+}^{\star}=-3 \tau^{1 / 2}+\mathcal{O}(\tau), \\
w_{1}(\tau) & =-\mathrm{d}_{\tau} \xi_{+}^{\star}(\tau)=-\frac{1}{2} \tau^{-1 / 2}+\mathcal{O}(1) .
\end{aligned}
$$

Notice that the linearization of (104) admits the eigenvalues $\alpha \pm \mathrm{i} \beta^{1 / 2}$. It can be diagonalised approximately by the transformation

$$
\zeta=\frac{1}{\sqrt{2}}\left[\mathrm{e}^{\mathrm{i} \pi / 4} \beta^{1 / 4}-\mathrm{e}^{-\mathrm{i} \pi / 4} \alpha \beta^{-1 / 4}\right] \xi_{1}+\frac{1}{\sqrt{2}} \mathrm{e}^{-\mathrm{i} \pi / 4} \beta^{-1 / 4} \eta_{1}
$$

We obtain a system similar to (56):

$$
\varepsilon \dot{\zeta}=\rho(\tau) \zeta+\varepsilon \varphi(\tau) \bar{\zeta}+\varepsilon w(\tau)+g(\zeta, \bar{\zeta}, \tau, \varepsilon),
$$

where

$$
\begin{aligned}
\rho(\tau) & =\alpha+\mathrm{i} \beta^{1 / 2}+\frac{1}{2} \mathrm{i} \varepsilon \dot{\alpha} \beta^{-1 / 2}, \\
\varphi(\tau) & =\frac{1}{4} \mathrm{i} \dot{\beta} \beta^{-1}-\frac{1}{2} \dot{\alpha} \beta^{-1 / 2}=\mathcal{O}\left(\tau^{-1}\right), \\
w(\tau) & =\frac{1}{\sqrt{2}} \mathrm{e}^{-\mathrm{i} \pi / 4} \beta^{-1 / 4} R(0,0, \tau, 0)+\mathcal{O}\left(\tau^{1 / 4}\right), \\
g(\zeta, \bar{\zeta}, \tau, \varepsilon) & =\mathcal{O}\left(\tau^{-1 / 4}|\zeta|^{2}\right)+\mathcal{O}\left(\tau^{-1}|\zeta|^{3}\right)+\mathcal{O}\left(\tau^{-5 / 4}|\zeta|^{4}\right) .
\end{aligned}
$$

Finally, by Corollary 5.9, we get the following estimate on the initial condition:

$$
\left|\zeta\left((\varepsilon / d)^{2 / 3}\right)\right| \leqslant M_{0} d^{1 / 2} \varepsilon^{1 / 2}
$$

The main result of this subsection is the following. 
Proposition 5.10. Assume that $c=\mathrm{d} a / \mathrm{d} \mu(0)<\frac{1}{2}$. Then there exist positive constants $M, \kappa$ and $T$ such that, if $\varepsilon$ and $d$ are sufficiently small, any solution of (107) with initial condition (109) satisfies the bounds

$$
|\zeta(\tau)| \leqslant M\left[\varepsilon \tau^{-3 / 4}+\varepsilon^{1 / 2} \mathrm{e}^{-\kappa \tau^{2} / \varepsilon}\right] \quad \text { for }(\varepsilon / d)^{1 / 3} \leqslant \tau \leqslant T .
$$

Corollary 5.11. On the same time interval, there is a constant $M_{1}>0$ such that the solution of (102) satisfies

$$
\begin{aligned}
\left|\xi(\tau)-\xi_{+}^{\star}(\tau)\right| & \leqslant M_{1}\left[\varepsilon \tau^{-1}+\varepsilon^{1 / 2} \tau^{-1 / 4} \mathrm{e}^{-\kappa \tau^{2} / \varepsilon}\right], \\
|\eta(\tau)| & \leqslant M_{1}\left[\varepsilon \tau^{-1 / 2}+\varepsilon^{1 / 2} \tau^{1 / 4} \mathrm{e}^{-\kappa \tau^{2} / \varepsilon}\right] .
\end{aligned}
$$

The proof of Proposition 5.10 follows directly from the two lemmas given below.

Lemma 5.12. Assume that $c<\frac{1}{2}$. There are positive constants $M$ and $T$ such that (107) admits a particular solution $\zeta_{0}(\tau)$ satisfying

$$
\left|\zeta_{0}(\tau)\right| \leqslant M \varepsilon \tau^{-3 / 4} \quad \text { for }(\varepsilon / d)^{1 / 3} \leqslant \tau \leqslant T .
$$

Proof: The proof is similar to the proof of Lemma 5.6, so we only outline the differences.

1. We have to eliminate quadratic terms from the equation as well. In order to get a normal form similar to (71), we start by eliminating quadratic terms, then we remove the term linear in $\bar{\zeta}$, and then only the nonresonant cubic terms.

2. For $(\varepsilon / d)^{1 / 3} \leqslant \tau \leqslant \varepsilon^{1 / 2}$, we eliminate the real part of the linear term by the transformation $\zeta=\exp \left[c \tau^{2} / \varepsilon\right] \zeta_{1}$. We then change the direction of time, fix a $\zeta_{1}\left(\varepsilon^{1 / 2}\right)$ of order $\varepsilon^{5 / 8}$ and use Lemma 5.6.

3. For $\tau \geqslant \varepsilon^{1 / 2}$, we proceed exactly as in Lemma 5.6.

Lemma 5.13. Any solution of (107) with initial condition (109) satisfies

$$
\left|\zeta(\tau)-\zeta_{0}(\tau)\right| \leqslant M \varepsilon^{1 / 2} \mathrm{e}^{-\kappa \tau^{2} / \varepsilon} \quad \text { for }(\varepsilon / d)^{1 / 3} \leqslant \tau \leqslant T
$$

ProOF:

- Step 1: Hamiltonian system.

Consider, as a special case of (104), the Hamiltonian system

$$
\begin{aligned}
& \varepsilon \dot{\xi}_{1}=\eta_{1}-\frac{1}{2} \varepsilon \tau^{-1 / 2} \\
& \varepsilon \dot{\eta}_{1}=-2 \tau \xi_{1}-3 \sqrt{\tau} \xi_{1}^{2}-\xi_{1}^{3} .
\end{aligned}
$$

Lemma 5.13 shows the existence of a particular solution $\xi_{0}(\tau)=\mathcal{O}\left(\varepsilon \tau^{-1}\right), \eta_{0}(\tau)=$ $\mathcal{O}\left(\varepsilon \tau^{-1 / 2}\right)$. If $\left(\xi_{1}, \eta_{1}\right)=\left(\xi_{0}, \eta_{0}\right)+\left(\xi_{2}, \eta_{2}\right)$, the dynamics of $\left(\xi_{2}, \eta_{2}\right)$ is governed by a Hamiltonian of the form

$$
H\left(\xi_{2}, \eta_{2}, \tau\right)=\frac{1}{2} \eta_{2}^{2}+\tau k_{1}(\tau) \xi_{2}^{2}+\sqrt{\tau} k_{2}(\tau) \xi_{2}^{3}+\frac{1}{4} \xi_{2}^{4},
$$

where $k_{1}(\tau)$ and $k_{2}(\tau)$ are bounded functions. Using (109), one can show that for sufficiently small $d$, there exists a constant $M_{0}$ such that

$$
H(\tau) \leqslant M_{0} d^{2} \tau^{2}
$$


- Step 2: Normal forms.

We write (107) in the form

$$
\varepsilon \dot{\zeta}=\rho(\tau) \zeta+\varepsilon \varphi(\tau) \bar{\zeta}+\varepsilon w(\tau)+g^{0}(\zeta, \bar{\zeta}, \tau)+g^{1}(\zeta, \bar{\zeta}, \tau, \varepsilon),
$$

where $g^{0}(\zeta, \bar{\zeta}, \tau)$ is the contribution of the Hamiltonian approximation (114) of (104), and

$$
g^{1}(\zeta, \bar{\zeta}, \tau, \varepsilon)=\mathcal{O}\left(\tau^{1 / 4}|\zeta|^{2}\right)+\mathcal{O}\left(\tau^{-1 / 2}|\zeta|^{3}\right)+\mathcal{O}\left(\tau^{-5 / 4}|\zeta|^{4}\right)
$$

This relation holds because the full system (104) is a perturbation of size $1+\sqrt{\tau}$ of the Hamiltonian system (114).

We now perform a number of changes of variables: a translation $\zeta=\zeta_{0}(\tau)+\zeta_{1}$, where $\zeta_{0}(\tau)$ is the particular solution of Lemma 5.12; a linear change of variables $z_{1}=\zeta_{2}+s(\tau) \bar{\zeta}_{2}$, where $s$ satisfies (67), which cancels the term linear in $\bar{\zeta}_{1}$; and a transformation to normal form $\zeta_{2}=\zeta_{3}+h\left(\zeta_{3}, \bar{\zeta}_{3}, \tau\right)$ which yields the equation

$$
\varepsilon \dot{\zeta}_{3}=\rho_{3}(\tau) \zeta_{3}+c^{0}(\tau ; \varepsilon)\left|\zeta_{3}\right|^{2} \zeta_{3}+c^{1}(\tau ; \varepsilon)\left|\zeta_{3}\right|^{2} \zeta_{3}+\mathcal{O}\left(\tau^{-5 / 4}\left|\zeta_{3}\right|^{4}\right),
$$

where the functions $c^{0}=\mathcal{O}\left(\tau^{-1}\right)$ and $c^{1}=\mathcal{O}\left(\tau^{-1 / 2}\right)$ denote contributions of $g^{0}$ and $g^{1}$, respectively.

- Step 3: Bounds on the coefficients.

We claim that

$$
\operatorname{Re} \rho_{3}(\tau)=\alpha(\tau)+\mathcal{O}\left(\varepsilon \tau^{-1 / 2}\right), \quad \operatorname{Re} c^{0}(\tau)=\mathcal{O}\left(\tau^{-1 / 2}\right) .
$$

The first claim can be checked by a direct calculation. We observe that the linearization of (104) around the particular solution $\left(\xi_{0}, \eta_{0}\right)$ has the form $\left(\begin{array}{cc}0 & 1 \\ -\tilde{\beta} & 2 \tilde{\alpha}\end{array}\right)$, where $\tilde{\alpha}=$ $\alpha+\mathcal{O}\left(\varepsilon \tau^{-1 / 2}\right)$ and $\tilde{\beta}=\beta+\mathcal{O}\left(\varepsilon \tau^{-1 / 2}\right)$. Then we show that the function $s(\tau)$ occuring in the linear transformation is such that $\operatorname{Im} s(\tau)=\mathcal{O}\left(\varepsilon \tau^{-1}\right)$.

The second claim can be proved without lengthy calculations. By construction, $\tau c^{0}(\tau)$ is polynomial in $\left\{\tau^{1 / 2}, \varepsilon \tau^{-3 / 2}\right\}$. If we assume by contradiction that the leading term of $\operatorname{Re} c^{0}(\tau)$ is of order $\tau^{-1}$, we reach the conclusion that $\zeta_{2}$ would grow faster than allowed by the estimate (116).

- Step 4: Final estimate.

The Lyapunov function $V=\left|z_{3}\right|^{2}$ satisfies the equation

$$
\varepsilon \dot{V} \leqslant-2 \kappa \tau V\left[1-M_{2} \tau^{-3 / 2} V-M_{2} \tau^{-9 / 4} V^{3 / 2}\right],
$$

where $\kappa=\frac{1}{2}-c$ and $M_{2}>0$. We obtain the conclusion in a similar way as in Proposition 5.8.

\section{Qualitative properties and robustness}

We conclude by discussing a few cases to which Theorem 2.2 does not apply.

- Other values of $\mathrm{d} a / \mathrm{d} \mu$ :

The result requires a sufficiently low value of $\mathrm{d} a / \mathrm{d} \mu$, which should be such that we traverse the bifurcation diagram Fig. 2 from region A to one of the regions D, E or F. Note that in a neighbourhood of the bifurcation point, the qualitative behaviour is 

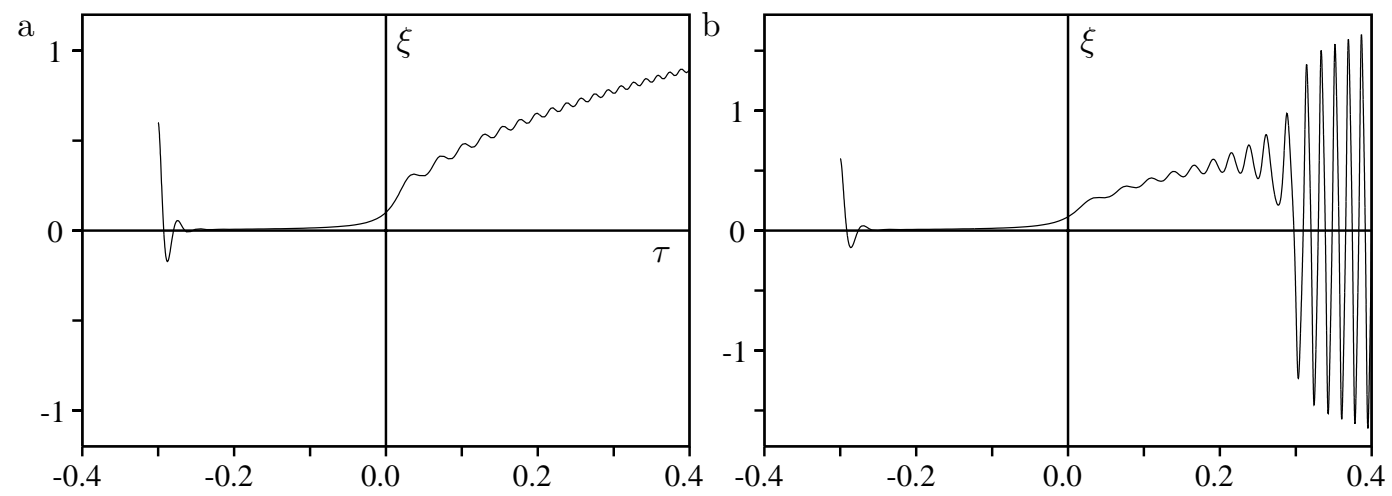

Figure 4. Same as Fig. 1, but for values of $\mathrm{d} a / \mathrm{d} \mu$ to which Theorem 2.2 does not apply. (a) When $\mu(\tau)=2 \tau$, we move just along the C-D line of Fig. 2; the equilibria are marginally stable, thus the solution keeps oscillating around them with a constant amplitude. (b) When $\mu(\tau)=0.5 \tau$, we go from region A to region $\mathrm{C}$; now the asymmetric equilibria are unstable; the trajectory oscillates for some time around them because of Proposition 5.8 , but ultimately escapes to the periodic orbit.

independent of the functions $\gamma$ and $\delta$. The only relevant fact is that the bifurcating equilibrium branches should be attracting. This fact is not surprising when we steer into regions $\mathrm{E}$ or $\mathrm{F}$. It is a bit more surprising when we go into region $\mathrm{D}$, because the equilibrium is surrounded by an unstable periodic orbit. The fact that the trajectory lands inside this orbit is due to the specific nature of the Hamiltonian approximation valid near the bifurcation point.

If we further increase $\mathrm{d} a / \mathrm{d} \mu$ so as to reach region $\mathrm{C}$, the equilibria are no longer attracting. On the C-D boundary, trajectories oscillate around these equilibria with approximately constant amplitude (Fig. Ma), while in the region C, they depart from them after a time of order $\varepsilon^{1 / 2}$ and finally reach the outer periodic orbit (Fig. đb). In view of our control problem, this behaviour is not desirable in the sense that we have an appearance of large amplitude oscillations, although the delay is not macroscopic.

- Imperfect control:

While constructing the feedback control in Section 4, we had to adjust precisely three parameters: the imaginary eigenvalue shift $\nu$ and the two parameters $v_{1}$ and $v_{2}$ of the nonlinear part. If these parameters are not set exactly to the desired value, the dynamics will still be governed by equation (11), but the functions $a(\tau), \mu(\tau), \gamma(\tau)$ and $\delta(\tau)$ will not vanish exactly at the same time. The same is true to some extent if the feedback $u(x, \tau)$ does not vanish exactly on the nominal equilibrium branch $x^{\star}(\tau)$. In other words, such imperfections result in the fact that we traverse the bifurcation diagram of Fig. 2 on a curve which misses the origin. Two situations may occur:

If we traverse the diagram on a path A-F-E, we first experience a pitchfork bifurcation, which results in an exchange of stabilities as shown in [LS]. Thus the trajectory will still track a stable equilibrium, but the bifurcation will occur a little bit earlier.

If we traverse the diagram on a path A-B-C, the situation is less favourable. The Hopf bifurcation A-B will not be felt immediately, but when the region $\mathrm{C}$ is reached, there is a risk that the trajectory jumps to the periodic orbit. This behaviour can only be avoided if the slope $\mathrm{d} \mu / \mathrm{d} a$ is large enough that the region $\mathrm{D}$ is reached before the trajectory has departed from the asymmetric branch. 


\section{References}

[Ab] E.H. Abed, Bifurcation-theoretic issues in the control of voltage collapse, in J.H. Chow (Ed.), Systems and control theory for systems (Springer, New York, 1995).

[Ae] D. Aeyels, Stabilization of a class of nonlinear systems by a smooth feedback control, Systems Control Lett. 5:289-294 (1985).

[Ben] E. Benoît (Ed.), Dynamic Bifurcations, Proceedings, Luminy 1990 (SpringerVerlag, Lecture Notes in Mathematics 1493, Berlin, 1991).

[B1] N. Berglund, Adiabatic Dynamical Systems and Hysteresis, Thesis EPFL no 1800 (1998). Available at http://dpwww.epfl.ch/instituts/ipt/berglund/these.html

[B2] N. Berglund, On the Reduction of Adiabatic Dynamical Systems near Equilibrium Curves, Proceedings of the International Workshop "Celestial Mechanics, Separatrix Splitting, Diffusion", Aussois, France, June 21-27, 1998.

[BK] N. Berglund, H. Kunz, Memory Effects and Scaling Laws in Slowly Driven Systems, J. Phys. A 32:15-39 (1999).

[Ca] J. Carr, Applications of Centre Manifold Theory (Springer-Verlag, New York, 1981).

[CHK] F. Colonius, G. Häckl, W. Kliemann, Controllability near a Hopf bifurcation, proc. 31st IEEE Conf. Dec. Contr., Tucson, Arizona (1992).

[CK] F. Colonius, W. Kliemann, Controllability and stabilization of one-dimensional systems near bifurcation points, Systems Control Lett. 24:87-95 (1995).

[Fe] N. Fenichel, Geometric singular perturbation theory for ordinary differential equations, J. Diff. Eq. 31:53-98 (1979).

[GH] J. Guckenheimer, P. Holmes, Nonlinear Oscillations, Dynamical Systems, and Bifurcations of Vector Fields (Springer-Verlag, New York, 1983).

[Hab] R. Haberman, Slowly varying jump and transition phenomena associated with algebraic bifurcation problems, SIAM J. Appl. Math. 37:69-106 (1979).

[HS] G. Hckl, K.R. Schneider, Controllability near Takens-Bogdanov points, J. Dynam. Control Systems 2:583-598 (1996).

[Hal] J.K. Hale, Ordinary differential equations (J. Wiley \& Sons, New York, 1969).

[KKR] A.I. Khibnik, B. Krauskopf, C. Rousseau, Global study of a family of cubic Liénard equations, Nonlinearity 11:1505-1519 (1998).

[Kr] S.G. Krein, Linear Differential Equations in Banach Spaces (American Mathematical Society, Providence, Rhode Island, 1971).

[LS] N.R. Lebovitz, R.J. Schaar, Exchange of Stabilities in Autonomous Systems I, II, Stud. in Appl. Math. 54:229-260 (1975). Stud. in Appl. Math. 56:1-50 (1977). 
[MS] W. Müller, K.R. Schneider, Feedback Stabilization of Nonlinear Discrete-Time Systems, J. Differ. Equations Appl. 4:579-596 (1998).

[Ne1] A.I. Neishtadt, Persistence of stability loss for dynamical bifurcations I, II, Diff. Equ. 23:1385-1391 (1987). Diff. Equ. 24:171-176 (1988).

[Ne2] A.I. Neishtadt, On Calculation of Stability Loss Delay Time for Dynamical Bifurcations in D. Jacobnitzer Ed., XI ${ }^{\text {th }}$ International Congress of Mathematical Physics (International Press, Boston, 1995).

[PR] L.S. Pontryagin, L.V. Rodygin, Approximate solution of a system of ordinary differential equations involving a small parameter in the derivatives, Dokl. Akad. Nauk SSSR 131:237-240 (1960).

[Sh] M.A. Shishkova, Examination of one system of differential equations with a small parameter in highest derivatives, Dokl. Akad. Nauk SSSR 209:576-579 (1973). [English transl.: Soviet Math. Dokl. 14:384-387 (1973)].

[Ta] F. Takens, Forced oscillations and bifurcations, Comm. Math. Inst., Rijksuniversiteit Utrecht 3:1-59 (1974).

[VBK] A.B. Vasil'eva, V.F. Butusov, L.V. Kalachev, The Boundary Function Method for Singular Perturbation Problems (SIAM, Philadelphia, 1995).

[VT] E.V. Volokitin, S.A. Treskov, Parametric portrait of the Fitz-Hugh differential system, Math. Modelling 6:65-78 (1994). (in Russian)

[Wa] W. Wasow, Asymptotic expansions for ordinary differential equations (Krieger, New York, 1965, 1976). 